\title{
Fair allocation of conflicting items
}

\author{
Halvard Hummel $^{1}$ (D) $\cdot$ Magnus Lie Hetland $^{1}$ (D)
}

Accepted: 18 November 2021 / Published online: 23 December 2021

(c) The Author(s) 2021

\begin{abstract}
We study fair allocation of indivisible items, where the items are furnished with a set of conflicts, and agents are not permitted to receive conflicting items. This kind of constraint captures, for example, participating in events that overlap in time, or taking on roles in the presence of conflicting interests. We demonstrate, both theoretically and experimentally, that fairness characterizations such as EF1, MMS and MNW still are applicable and useful under item conflicts. Among other existence, non-existence and computability results, we show that a $1 / \Delta$-approximate MMS allocation for $n$ agents may be found in polynomial time when $n>\Delta>2$, for any conflict graph with maximum degree $\Delta$, and that, if $n>\Delta$, a 1/3-approximate MMS allocation always exists.
\end{abstract}

Keywords Fair allocation · Graph coloring · Approximation

\section{Introduction}

We are interested in the problem of allocating a set of indivisible items among a set of agents with additive valuations, and beyond finding an efficient solution, where the total utility is high, we want the allocation to be fair, in some sense-a problem that has been studied extensively in the last couple of decades [8]. More recently, variations of this problem have appeared, where the bundles of items allocated must conform to some constraints, meaning that not all allocations are feasible. For example, if the items are structured as a matroid, one may require that the set of all allocated items form a basis [18], or that each bundle be an independent set of the matroid [4]. One might also partition the items into different categories, and limit each agent to a certain number from each [4]. Or the items may be the nodes of a graph, where a natural requirement is for each bundle to be connected [7].

In this paper, we look at yet another form of constraint, where items may be in conflict with each other, and an agent may receive at most one of any two conflicting items. This is a situation that may occur in many realistic allocation scenarios. For example, such conflicts arise naturally in scheduling problems where the items represent participation in

Halvard Hummel

halvard.hummel@ntnu.no

Magnus Lie Hetland

mlh@ntnu.no

1 Norwegian University of Science and Technology, Trondheim, Norway 
some activities—-such as conference sessions or panels at a convention-with limited seating. The activities are associated with time intervals, and no agent may participate in two of them simultaneously. Thus when participants register for a prioritized subset, any fair allocation must take care to avoid scheduling conflicts. In another scenario, the items may represent sought-after administrative positions in an academic institution, where the conflicts are conflicts of interest. Positions should be allocated fairly among qualified applicants, without anyone appointed to two positions where one has the power to approve proposals submitted by the other, for example.

Our contributions. We study the problem of fairly allocating conflicting items, and present several new results in this setting. We map out, both in broad strokes and for certain special cases, to what extent envy-freeness up to one item (EF1) is guaranteed to exist, as well as when it is guaranteed by maximum Nash welfare (MNW), and when it may be achieved in polynomial time (Sect. 3.1). We adapt the randomized graph coloring procedure of Pemmaraju and Srinivasan [30], which is expected to approximate any individual agent's proportional share to within a factor of $1-1 / e$, and guarantees that for large instances of certain kinds, the deterministic proportionality guarantee does not fall far below this expectation (Sect. 3.2). We then present a series of results on maximin share (MMS) allocation with item conflicts (Sect. 3.3), with the main results being that, if the maximum degree $\Delta$ of the conflict graph is lower than the number of agents, ${ }^{1}$ (i) there exists an $\alpha$-approximate MMS allocation, with $\alpha>1 / 3$ (Theorem 1); and (ii) an $\alpha$-approximate MMS allocation may be found in polynomial time, with $\alpha>1 / \Delta$ when $\Delta>2$ (Theorem 2). Finally, we examine the behavior of various fairness measures in practice, that is, empirically studying how random allocation, EF1, MMS, proportionality and MNW are affected by the introduction of item conflicts, on 18,629 randomly generated graphs (Sect. 4), with the main conclusion being that fairness is largely unharmed, with random allocation improving as an MMS approximation, all instances exhibiting EF1 and MMS, and MNW producing EF1 in almost all cases, with a tight approximation of MMS.

Prior work. Fair allocation of conflicting items has been studied by Chiarelli et al. [13], who considered partial egalitarian (maximin) allocations, i.e., allocations that maximize the value the worst-off agent receives, but where some items may remain unallocated. In this paper, we study conventional (complete) allocations, and focus on other fairness criteria such as envy-freeness, maximin share guarantees and Nash welfare. ${ }^{2}$ The relation between our scenario and that of Chiarelli et al. depends on the relationship of the maximum degree of the conflict graph, $\Delta$, and the number of agents, $n$. For the maximin criterion used by Chiarelli et al., there is no difference between allowing and disallowing partial allocations when $n>\Delta$, which is also a precondition for several of our results. Conversely, the hardness results of Chiarelli et al. rely on graphs where $\Delta>n$, which means they do not contradict our approximability result for MMS (Theorem 2). Without the fairness aspect, allocation with item conflicts reduces to the much-studied problem of graph coloring [24], with each item becoming a node, and each color an agent. Some work has already been done on equity in graph coloring [25], but links to the burgeoning field of fair item allocation seem so far to be missing from the literature.

\footnotetext{
1 This restriction ensures that any partial allocation may be completed without reassigning any items.

${ }^{2}$ Requiring complete allocations is necessary for some fairness criteria (e.g., EF1) to make sense, and may be required in real-world allocation scenarios.
} 


\section{Preliminaries}

We study the problem of fairly allocating a set of items among a set of agents, where certain pairs of items are not allowed together. We call this problem fair allocation of conflicting items. In the following, for $k \in \mathbb{Z}^{+},[k]$ denotes the set $\{1,2, \ldots, k\}$.

Definition 1 An instance of the fair allocation of conflicting items problem, or more concisely, a problem instance, is a quadruple $(N, M, V, G)$, where

- $\quad N$ is a set of $n$ agents;

- $M$ is a set of $m$ items;

- $V$ is a family of $n$ valuation functions, $v_{i}: 2^{M} \rightarrow \mathbb{R}_{\geq 0}$; and

- $G=(M, E)$ is an undirected conflict graph.

Unless otherwise stated, we assume $N=[n]$ and $M=[m] .^{3}$

We say that two neighboring items in the conflict graph are conflicting items or, equivalently, in conflict. Although more general valuations are possible, we shall assume that all valuation functions are additive. For simplicity, we let both $v_{i j}$ and $v_{i}(j)$ denote $v_{i}(\{j\})$.

For an instance of the fair allocation of conflicting items problem, an allocation, $A=\left\langle A_{1}, A_{2}, \ldots, A_{|N|}\right\rangle$, is an $|N|$-partition of the set of items, assigning set $A_{i}$ to agent $i$. A set of items $A_{i} \in A$ is called a bundle. An $|N|$-partition of a strict subset of the items is called a partial allocation. An allocation that is not partial is complete.

We wish to find feasible allocations that are as fair as possible. An allocation is said to be feasible if no bundle contains a pair of conflicting items. Note that any feasible allocation forms an $|N|$-coloring of the conflict graph and that each bundle in a feasible allocation is an independent set in the conflict graph. What constitutes a fair allocation is less clearcut, and many characterizations exist. In this paper, we consider the four fairness criteria of maximum Nash welfare, envy-freeness up to one good, proportionality and maximin share guarantee.

The Nash social welfare function, or Nash welfare, is similar to a plain utilitarian welfare, except that individual utilities are multiplied to produce an aggregate. For allocation without conflicts, maximizing the Nash welfare leads to a good tradeoff between fairness and efficiency and guarantees fulfillment or close approximation of several other fairness criteria [12].

Definition 2 For a problem instance $(N, M, V, G)$, the Nash welfare (NW) of a feasible allocation $A$ is given by

$$
\mathrm{NW}(A)=\left(\prod_{i \in N} v_{i}\left(A_{i}\right)\right)^{1 / n} .
$$

$A$ is said to be a maximum Nash welfare (MNW) allocation if there is no feasible allocation with a higher Nash welfare.

\footnotetext{
${ }^{3}$ An exception is reduced instances where agents or items have been removed (cf. Sect. 3.3).
} 
Envy-freeness is a very natural criterion for fair allocation, which requires that no agent be envious of any other agent. Envy-freeness is often unobtainable when considering indivisible items. This is easily seen when allocating a single item to two agents. Instead of full envy-freeness, we consider the relaxation to envy-freeness up to one good, introduced by Budish [11]. This fairness criterion instead requires that for any pair of agents $i$ and $i^{\prime}$, the bundle $A_{i^{\prime}}$ contains an item so that $i$ would not envy $i^{\prime}$ if the item was removed from $A_{i^{\prime}}$. More formally:

Definition 3 For a problem instance $(N, M, V, G)$, a feasible allocation $A$ is said to be envy-free up to one good (EF1) if for all $i, i^{\prime} \in N$, where $A_{i^{\prime}} \neq \emptyset$,

$$
v_{i}\left(A_{i}\right) \geq v_{i}\left(A_{i^{\prime}}\right)-\max _{j \in A_{i^{\prime}}} v_{i j} .
$$

A different relaxation of envy-freeness is proportionality, where agents should receive at least their subjective fair of the total value available. More formally:

Definition 4 For a problem instance $(N, M, V, G)$, a feasible allocation $A$ is called proportional if each agent $i$ assigns a value of at least $v_{i}(M) /|N|$ to its bundle.

Maximin share guarantee is another fairness criterion introduced by Budish [11]. Here, we want to guarantee each agent a bundle valued at no less than what the agent would have been guaranteed if it were to create a feasible allocation, but had to choose its own bundle last.

Definition 5 For a problem instance $I=(N, M, V, G)$, an agent $i$ 's maximin share (MMS) is given by

$$
\mu_{i}^{I}=\max _{A \in \mathcal{F}} \min _{A_{j} \in A} v_{i}\left(A_{j}\right)
$$

where $\mathcal{F}$ is the set of all feasible allocations of $I$. If the instance $I$ is obvious from context, we omit it and write $\mu_{i}$.

A feasible allocation $A$ with $\min _{A_{j} \in A} v_{i}\left(A_{j}\right)=\mu_{i}^{I}$ for agent $i$ is said to be an MMS partition of $I$ for $i$. A feasible allocation where each agent $i$ receives a bundle it values at no less than $\mu_{i}$, is called an MMS allocation. Even without conflicts, there are instances for which no MMS allocation exists [23]. Additionally, as calculating the MMS of an agent is NP-hard [33], finding MMS allocations is generally infeasible. Instead, approximations are usually considered. We say that a feasible allocation is $\alpha$-approximate $M M S$ if each agent receives a bundle they value at no less than $\alpha \mu_{i}$.

Several useful properties of MMS have been found in the ordinary, conflict-free setting. Many of these are not easily extendable or applicable to allocation under item conflicts, as will be discussed later; However, the basic properties of scale-freeness and normalization may be quite naturally extended to the new scenario. These properties simplify working with approximations of MMS, especially when finding polynomialtime algorithms. The proofs of the properties carry over from unconstrained allocation, and have been omitted. See, e.g., the works of Amanatidis et al. [2] and Garg et al. [16] for details. 
Lemma 1 (Scale-freeness) For a problem instance $I=(N, M, V, G)$, let $I^{\prime}=\left(N, M, V^{\prime}, G\right)$ be the problem instance where the valuations of each agent $i$ are scaled by some constant $c_{i}>0$. Then $\mu_{i}^{I^{\prime}}=c_{i} \mu_{i}^{I}$ and all MMS allocations and MMS partitions of I are also MMS allocations and MMS partitions of $I^{\prime}$.

Lemma 2 (Normalization) If $v_{i}(M)=|N|$ for an agent $i$ in a problem instance $I=(N, M, V, G)$, then $\mu_{i}^{I} \leq 1$.

Fair allocation of conflicting items is a generalization of fair allocation without conflicts, which is the special case of $G=(M, \emptyset)$. A similar problem to fair allocation of conflicting items, is fair allocation under cardinality constraints [4]. In this version of the problem, the items are divided into categories and a bundle may not contain more items from a single category than some given threshold. Instances of this version of the problem where each category has a threshold of 1 may be reduced directly to fair allocation of conflicting items, with the conflict graph becoming a collection of cliques, one per category.

While we only consider additive valuation functions in our instances, there exists research on other classes of valuation functions. Some of our results rely on reduction to unconstrained fair allocation with more complex valuation functions in order to maintain the conflicts to a certain degree. A function $f$ is fractionally subadditive (XOS) if there exists a finite set $F$ of additive functions such that for any set $S, f(S)=\max _{f^{\prime} \in F} f^{\prime}(S)$. Submodularity is a more restricted case. For a submodular function $f$ and any two sets $S$ and $S^{\prime}$, $f(S)+f\left(S^{\prime}\right) \geq f\left(S \cup S^{\prime}\right)+f\left(S \cap S^{\prime}\right)$.

A graph $G=(M, E)$ is complete if all vertices are neighbors, and empty if $E=\emptyset$. Given a set of vertices $S \subseteq M$, we let $G[S]$ denote the induced subgraph of $S$ on $G$, i.e., the graph consisting of the vertices in $S$ and all edges in $E$ that connect pairs of vertices in $S$. We let $\Delta(G)$ denote the maximum degree of the graph $G$, and $\mathcal{C}(G)$ the cardinality of its largest connected component. A $k$-coloring of $G$ is a coloring of the vertices in $G$ using $k$ distinct colors, such that no two neighboring vertices share a color. We let $\chi(G)$ denote the smallest integer $k$ for which a $k$-coloring exists for $G$. Note that the problem of determining if a graph is $k$-colorable is NP-complete. However, a $(\Delta(G)+1)$-coloring always exists and can greedily be found in polynomial time.

\section{Conflicting items in theory}

We are interested in determining, theoretically, to what extent we can guarantee the agents either fulfillment or approximation of various fairness criteria when there are conflicting items. Besides theoretical results, we are interested in the degree to which such fairness can be achieved in polynomial time. In this section, we explore the existence and non-existence of EF1, both by itself and as a product of MNW. We also explore approximations to both proportionality and MMS.

\subsection{Envy-freeness up to one good}

Without item conflicts, we know that EF1 allocations always exist [26]. With item conflicts, this is not always the case. The simplest example is when there are no feasible allocations at all, let alone EF1 allocations. We are more interested in cases where the items may, in fact, be allocated - but even then there are instances that do not admit an EF1 allocation. 
The following proposition shows that if an item is in conflict with as many items as there are agents, there always exists a set of binary valuation functions that precludes EF1, even if feasible allocations exist.

Proposition 1 For any graph $G=(M, E)$ with $\Delta(G) \geq n$, there is a problem instance $([n], M, V, G)$ that has no EF1 allocation.

Proof First of all, the instance may be unfeasible, i.e., it may have no feasible allocation. This would be the case, for example, for the complete graph $K_{n+1}$. Assume that there is some feasible allocation, and select some item $j$ with degree at least $n$. For every agent, let the neighbors of $j$ get a value of 1 , and let all other nodes get a value of 0 . Some agent $i$ must receive item $j$, and some agent $i^{\prime}$ must receive at least two of its neighbors, and $i$ will still envy $i^{\prime}$ after removing one of the items allocated to $i^{\prime}$, which means that no allocation for these valuations can be EF1.

In other words, an EF1 allocation is not guaranteed when the number of agents is no higher than the maximum degree of the graph. This is quite natural, as a high degree in relation to the number of agents limits the number of feasible allocations quite drastically. In the other direction, it is possible to show that for graphs with sufficiently small components, EF1 allocations always exist.

Proposition 2 For any graph $G=(M, E)$ with $\mathcal{C}(G) \leq n$, all problem instances $([n], M, V, G)$ have EF1 allocations that can be found in polynomial time.

Proof Let $I$ be the original instance, with conflicting items. We construct an instance $I^{\prime}$ with cardinality constraints by introducing one category $C_{h}$ for each connected component, consisting of its vertices, and setting the corresponding threshold to 1, i.e., no agent can get two items from the same category/connected component. Then $I$ is a relaxation of $I^{\prime}$, and any feasible allocation for $I^{\prime}$ is feasible for $I$. Biswas and Barman showed that there exists a polynomial-time algorithm that for any problem instance with cardinality constraints and additive valuation functions finds an EF1 allocation [4]. Using their algorithm, an EF1 allocation can be found for $I^{\prime}$ and consequently one can be found for $I$ as well.

Propositions 1 and 2 establish the existence and non-existence of EF1 allocations at opposite sides of a spectrum, in a sense, and leave open the question of whether EF1 exists when $\Delta(G)<n<\mathcal{C}(G)$. At least for some small subset of such instances, it can be shown that no $\mathrm{EF} 1$ allocations exist, as illustrated by the following example.

Example 1 Let $G=K_{3,3}$ (see below) and let there be a total of 4 agents. Then we have $\Delta(G)=3<4=n<\mathcal{C}(G)$. For all agents, let items 1,2 and 3 have a value of 2 and items 4 , 5 and 6 have a value of 3 . 


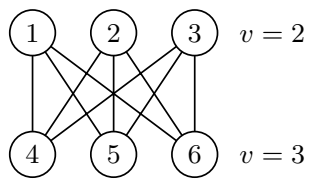

Either (i) one agent receives a bundle with two or more items worth 3 , or (ii) three agents receive a bundle with one item worth 3 . In the first case, we cannot guarantee the worst-off agent a bundle worth more than 2 . Since there is a bundle with at least two items worth 3, this does not allow for EF1. In the latter case, the last agent receives all items worth 2. After removing any item from this bundle, the value remains 4, which is more than any other agent receives. Consequently, an EF1 allocation does not exist.

Remark 1 Interestingly, it can be shown that the instance in Example 1 contains the fewest number of items for which there is no EF1 allocation when $n>\Delta(G)$. For $n=2$, this follows directly from Proposition 2. When $n \geq 3$ an EF1 allocation must always exist in this situation when $m \leq n+1$, as each agent can in turn choose their most-valuable remaining item until either no more remain or each agent has received one item. If $m=n+1$, the last item can be given to any agent without a conflicting item. Specifically for three agents, one can show, by working through the possible cases, that all such instances with five items admit an EF1 allocation.

The instance in Example 1 is not an isolated case. We can in fact find similar instances for any number of agents greater than three.

Proposition 3 For any $n \geq 4$, there exists a graph $G$ with $n>\Delta(G)$ and a set of valuations $V$, so that the instance $([n], M, V, G)$ does not admit any EF1 allocations.

Proof Let the graph $G$ be the complete bipartite graph $K_{n-1, n-1}$. This graph contains two partite sets of $n-1$ vertices, where there are no edges between vertices in the same set. For each pair of vertices in different sets, there is an edge between them. This results in the graph being regular, with a degree of $n-1$, as each vertex is connected to $n-1$ other vertices. If we use this graph as a conflict graph, each agent may only receive items from the same partite set.

For all agents, let the items in one of the sets each have a value of 2 , and the items in the other set each have a value of $2 n-5$. Any feasible allocation must contain either (i) a bundle with two or more items worth $2 n-5$ or (ii) $n-1$ bundles with a single item worth $2 n-5$.

In the first case, all other bundles must contain a value of at least $2 n-5$ for EF1 to be possible (more if a bundle has 3 or more items worth $2 n-5$ ). Each bundle must therefore contain at least one item, and at least two bundles must contain items only from the set where each item is valued 2 . The total value of the items worth 2 is $2(n-1)$, allowing the worst-off agent to receive a value of at most $n-1$. When $n>4,2 n-5>n-1$ and this case does not admit an EF1 allocation. For $n=4$, there are three items worth 2, and thus one of the two agents cannot receive a value of more than $2<2 n-5$.

In the second case, the last agent must receive all items worth 2 and has a bundle worth $2 n-2$. Removing any item from the bundle, results in a bundle valued at $2 n-4>2 n-5$. 
Meaning that all other agents envy the bundle, even after removing an item. Consequently, an EF1 allocation cannot exist.

Remark 1 and Proposition 3 show that when $\Delta(G)<n<\mathcal{C}(G)$, for $n \geq 4$, there are some instances for which EF1 exists, and some for which it does not. Giving a more detailed classification or characterization of such instances remains an open problem.

The instances used in Example 1 and Proposition 3 rely on very specific valuations for the items in order to show the non-existence of EF1. Changing the valuations slightly may easily result in the existence of EF1 allocations. Limiting the possible values each item can take, allows for some special cases where we have existence proofs and polynomial-time algorithms.

Proposition 4 If a problem instance $(N, M, V, G)$, where $|N|>2$, has valuation functions $v_{i}: M \rightarrow\{0,1\}$, and the components of $G$ are paths, then the instance has an EF1 allocation, which may be found in polynomial time.

Proof If there exists an item $j$ with $v_{i j}=0$ for each agent $i \in N$, who receives $j$ does not affect if an allocation is EF1, except to the extent that it may limit possible feasible allocations. However, because $|N|>2 \geq \Delta(G)$, for any feasible partial allocation of $M \backslash\{j\}$, there exists at least one agent with no conflicting items to $j$. In practice, this means that all items of this type can initially be removed and then, later, allocated arbitrarily. Thus, we only need to consider instances where there are no such items.

In order to show that an EF1 allocation exists for all instances where no item is valued at 0 by all agents, we will create a polynomial-time algorithm that iteratively allocates items by considering the connected components of $G$ one by one in arbitrary order. For each component $C$, iteratively allocating the items in order-from one end of the path to the other-while maintaining the following three properties.

(i) The allocation is EF1.

(ii) There exists an ordering, $\mathcal{O}$, of the agents such that no agent envies earlier agents in $\mathcal{O}$.

(iii) If some, but not all, items in the currently considered component, $C$, are unallocated, the agent $i$ that received the last allocated item in $C$ does not envy any other agent.

For property (ii), we rely on maintain an acyclic envy graph, i.e., a directed graph of the agents, with an edge from agent $i$ to agent $i^{\prime}$ if $i$ envies the bundle of $i^{\prime}$. When there are no cycles in the envy graph, any topological ordering of the graph orders the agents so that no agent envies earlier agents. We can easily find a topological ordering in polynomial time for an acyclic graph. Lipton et al. [26] give a polynomial-time procedure that decycles an envy graph, without breaking EF1 (property (i)), by exchanging bundles along the graph's cycles. Note that the procedure of Lipton et al. does not swap the bundles of agents that prior to the application of the decycling procedure did not envy any other agent. Since agent $i$ from (iii) is an agent of this type, the decycling procedure does not change $i$ 's bundle. As the content of the other bundles does not change, only their owners, agent $i$ will remain unenvious of all other agents also after applying the decycling procedure. Consequently, if (i) and (iii) hold prior to application of the decycling procedure, they also hold afterwards. 
Our algorithm starts with an initially empty allocation, for which all three properties hold. Then, in each step let $C$ be the current component, $\mathcal{O}$ any ordering that fulfills the requirements in (ii) and $i$ the agent from (iii). Also, let $j$ be the next unallocated item in $C$ and $j^{\prime}$ the (unallocated) item following $j$, if any exists. (If $j$ is the first item in $C$, then there is no agent $i$.) Additionally, let $i^{\prime}$ be the first agent in $\mathcal{O}$ with $i^{\prime} \neq i$ and $v_{i^{\prime}}(j)=1$, if any exists. Since each item is valued at 1 by at least one agent, $i^{\prime}$ always exists when $i$ does not. Then we allocate item $j$ and, possibly, $j^{\prime}$ by the following rules:

1. If $i^{\prime}$ exists, then allocate $j$ to $i^{\prime}$.

2. If $i^{\prime}$ and $j^{\prime}$ do not exist, then allocate $j$ to the first agent $i^{\prime \prime} \in \mathcal{O}, i^{\prime \prime} \neq i$.

3. If $i^{\prime}$ does not exist and $j^{\prime}$ exists, then allocate $j^{\prime}$ to the first agent $i^{\prime \prime \prime} \in \mathcal{O}$ with $v_{i^{\prime \prime \prime}}\left(j^{\prime}\right)=1$ and $j$ to any $i^{\prime \prime} \in N$ with $i^{\prime \prime} \neq i^{\prime \prime \prime}$ and $i^{\prime \prime} \neq i .^{4}$

We must now show that in each of these three cases, if (i), (ii) and (iii) hold prior to the allocation, then they hold afterwards as well. As seen earlier, property (ii) can be achieved by simply using the procedure of Lipton et al. after the allocations. Thus, we need only show that after applying the rules, but before using the decycling procedure of Lipton et al., (i) and (iii) hold. Let $A=\left\langle A_{1}, A_{2}, \ldots, A_{|N|}\right\rangle$ be the allocation prior to giving away $j$ and, possibly, $j^{\prime}$ in cases 1,2 and 3 , and $A^{\prime}=\left\langle A_{1}^{\prime}, A_{2}^{\prime}, \ldots, A_{|N|}^{\prime}\right\rangle$ the allocation afterwards.

Case 1: The only bundle that changes is $i^{\prime \prime}$ s bundle. That is $A_{i^{\prime}}^{\prime}=A_{i^{\prime}} \cup\{j\}$. For any agent $i^{*} \in N, i^{*} \neq i^{\prime}$ we know that either $v_{i^{*}}\left(A_{i^{*}}\right) \geq v_{i^{*}}\left(A_{i^{\prime}}\right)$ or $v_{i^{*}}(j)=0$. Otherwise, $i^{*}$ would have appeared before $i^{\prime}$ in $\mathcal{O}$ and been chosen instead of $i^{\prime}$. For any $i^{*}$ with $v_{i^{*}}\left(A_{i^{*}}\right) \geq v_{i^{*}}\left(A_{i^{\prime}}\right)$ we have $v_{i^{*}}\left(A_{i^{*}}^{\prime}\right) \geq v_{i^{*}}\left(A_{i^{\prime}}\right)=v_{i^{*}}\left(A_{i^{\prime}}^{\prime}\right)-v_{i^{*}}(j)$. Thus, since $v_{i^{*}}\left(A_{i^{\prime}}^{\prime}\right)=v_{i^{*}}\left(A_{i^{\prime}}\right)$ for all other $i^{*}$, $A^{\prime}$ is EF1 and (i) holds for $A^{\prime}$. Additionally, with binary valuations each item is valued at either 0 or 1 and combined with EF1 this implies that $v_{i^{\prime}}\left(A_{i^{\prime}}\right) \geq v_{i^{\prime}}\left(A_{i^{*}}\right)+1$ for all $i^{*} \in N$, $i^{*} \neq i^{\prime}$. As a result, we have

$$
v_{i^{\prime}}\left(A_{i^{\prime}}^{\prime}\right)=v_{i^{\prime}}\left(A_{i^{\prime}}\right)+v_{i^{\prime} j} \geq v_{i^{\prime}}\left(A_{i^{*}}\right)=v_{i^{\prime}}\left(A_{i^{*}}^{\prime}\right),
$$

and (iii) holds for $A^{\prime}$.

Case 2: The only bundle that changes is $i^{\prime \prime}$ 's bundle. That is $A_{i^{\prime \prime}}^{\prime}=A_{i^{\prime \prime}} \cup\{j\}$. Since the only agent that values $j$ at 1 is $i$, the only way that EF1 can be broken in $A^{\prime}$ is if $i$ is envious of $i^{\prime \prime}$. However, since (iii) holds for $A, v_{i}\left(A_{i}\right) \geq v_{i}\left(A_{i^{\prime \prime}}\right)$ and we have $v_{i}\left(A_{i}^{\prime}\right)=v_{i}\left(A_{i}\right) \geq v_{i}\left(A_{i^{\prime \prime}}\right)=v_{i}\left(A_{i^{\prime \prime}}^{\prime}\right)-v_{i j}$. Hence $A^{\prime}$ is EF1 and (i) holds for $A^{\prime}$. Since $j$ is the last item in $C$, the current component does not have any unallocated items and the next component, if any, has no allocated items. Thus, (iii) holds for $A^{\prime}$.

Case 3: The bundles of $i^{\prime \prime}$ and $i^{\prime \prime \prime}$ both change. That is $A_{i^{\prime \prime}}^{\prime}=A_{i^{\prime \prime}} \cup\{j\}$ and $A_{i^{\prime \prime \prime}}^{\prime}=A_{i^{\prime \prime \prime}} \cup\left\{j^{\prime}\right\}$. Since $i$ is the only agent that values $j$ at 1 , the changes to $i^{\prime \prime \prime}$ s bundle cannot, by the same logic as in case 2, result in $A^{\prime}$ not being EF1. Similarly, the changes to the bundle of $i^{\prime \prime \prime}$ are equivalent to the changes to $i^{\prime}$ 's bundle in case 1 (while the item differs, the properties of the change are the same). Thus, $A^{\prime}$ is EF1 and (i) holds for $A^{\prime}$. For (iii) there are two possibilities, if $i^{\prime \prime \prime} \neq i$, then $v_{i^{\prime \prime \prime}}(j)=0$ and $v_{i^{\prime \prime \prime}}\left(A_{i^{\prime \prime}}^{\prime}\right)=v_{i^{\prime \prime \prime}}\left(A_{i^{\prime \prime}}\right)$. Thus, due to binary valuations and $A$ being EF1, we have (as in case 1)

$$
v_{i^{\prime \prime \prime}}\left(A_{i^{\prime \prime \prime}}^{\prime}\right)=v_{i^{\prime \prime \prime}}\left(A_{i^{\prime \prime \prime}}\right)+v_{i^{\prime \prime \prime}}\left(j^{\prime}\right) \geq v_{i^{\prime \prime \prime}}\left(A_{i^{*}}\right)=v_{i^{\prime \prime \prime}}\left(A_{i^{*}}^{\prime}\right),
$$

${ }^{4}$ An agent $i^{\prime \prime \prime}$ always exists, as each item is valued at 1 by at least one agent. 
Fig. 1 The graph classes $P_{3}$ and $\overline{P_{3}}$

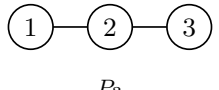

$P_{3}$
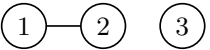

$\overline{P_{3}}$

for all $i^{*} \in N, i^{*} \neq i^{\prime \prime \prime}$, and (iii) holds for $A^{\prime}$ when $i^{\prime \prime \prime} \neq i$. If $i^{\prime \prime \prime}=i$, then we have that $v_{i}\left(A_{i^{\prime \prime}}^{\prime}\right)=v_{i}\left(A_{i^{\prime \prime}}^{\prime}\right)+1$. In which case the step $v_{i^{\prime \prime \prime}}\left(A_{i^{*}}\right)=v_{i^{\prime \prime \prime}}\left(A_{i^{*}}^{\prime}\right)$ from the above equation does not hold when $i^{*}=i^{\prime \prime}$. Since $v_{i}\left(A_{i}\right) \geq v_{i}\left(A_{i^{\prime \prime}}\right)$ by (iii), the following equation holds

$$
v_{i}\left(A_{i}^{\prime}\right)=v_{i}\left(A_{i}\right)+v_{i}\left(j^{\prime}\right) \geq v_{i}\left(A_{i^{\prime \prime}}\right)+v_{i}(j)=v_{i}\left(A_{i^{\prime \prime}}^{\prime}\right),
$$

and (iii) also holds for $A^{\prime}$ when $i^{\prime \prime \prime}=i$.

It can easily be verified that the three cases cover all possible situations and that no agent receives two conflicting items. Since all three properties hold before and after each step and all items are eventually given away, the algorithm produces complete EF1 allocations. It can also easily be verified that each of the described steps can be performed in polynomial time in the number of agents and items. Since the number of steps is bounded by the number of items, the algorithm runs in polynomial time.

As mentioned in Sect. 2, valid allocations for a given conflict graph $G$ are merely the $n$-colorings of $G$, and quite a lot of work has been done on certain forms of fairness in the graph coloring domain. In particular, a coloring is said to be equitable if the number of vertices colored by any two colors differ by at most one. For identical valuations, this is equivalent to EF1. Lih [25] provides an overview of many results of the equitable coloring problem for various graph classes, where the focus is on minimizing the number of colors. In our setting, however, the number of colors is given, and we have already seen in Proposition 1 that we need $n>\Delta(G)$ for all conflict graphs to have EF1 instances. In this case, we are guaranteed an equitable coloring, or, equivalently, an EF1 allocation for identical values..$^{5}$

Proposition 5 If a problem instance $(N, M, V, G)$, where $|N|>\Delta(G)$, has identical values, i.e., $v_{i j}=v_{i^{\prime} j^{\prime}}$ for $i, i^{\prime} \in N, j, j^{\prime} \in M$, then the instance has an EF1 allocation, which may be found in polynomial time.

In the context of equitable coloring, this is a well-known result, originating as a conjecture of Erdős. See, e.g., the 2008 paper by Kierstead and Kostochka [21] for some of its history, as well as a simplified proof and corresponding algorithm.

An interesting result for unconstrained allocation is that MNW leads to EF1 [12]. With conflicting items, we have seen that there are instances that do not admit an EF1 allocation, despite there being many feasible allocations. Consequently, we cannot guarantee that MNW leads to EF1 in this setting. Even so, it may be useful to determine whether MNW leads to EF1 when EF1 allocations do exist. This is not the case, as can be seen in the following proposition.

\footnotetext{
${ }^{5}$ Note also that for identical values, an EF1 solution will be MNW, and if EF1 exists, all MNW solutions are EF1.
} 
Fig. 2 Valuations for two agents, where $\mathrm{MNW}$ is not $\mathrm{EF} 1$, for $G=P_{3}\left(\right.$ left) and $G=\overline{P_{3}}$

\begin{tabular}{cccc}
\hline$i$ & $v_{i 1}$ & $v_{i 2}$ & $v_{i 3}$ \\
\hline 1 & 2 & 2 & 3 \\
2 & 6 & 5 & 6 \\
\hline
\end{tabular}

\begin{tabular}{cccc}
\hline$i$ & $v_{i 1}$ & $v_{i 2}$ & $v_{i 3}$ \\
\hline 1 & 2 & 1 & 3 \\
2 & 6 & 5 & 6 \\
\hline
\end{tabular}

Proposition 6 If the graph $G=(M, E)$ is complete or empty, then for any problem instance $([n], M, V, G)$ with $n>\Delta(G)$, all MNW allocations are EFI. If $G$ is neither complete nor empty, there is a problem instance $([n], M, V, G)$ with $n \geq \Delta(G)$ for which there exists at least one EF1 allocation, but for which no MNW allocation is EF1.

Proof First of all, if $G=K_{k}$ for some $k \leq n$, each bundle can only contain at most one item. Obviously, all allocations must then be EF1. Note that the case of $k>n$ has no feasible allocations. When $E=\emptyset$, this is equivalent to the fair allocation problem without conflicts. For this problem, Caragiannis et al. [12] showed that all MNW allocations are EF1.

If neither $G=K_{k}$ nor $E=\emptyset$, then $G$ contains at least one subset of three vertices that form an induced subgraph of either $P_{3}$ or $\overline{P_{3}}$ (see Fig. 1). If $G=P_{3}$ or $G=\overline{P_{3}}$, with two agents, the valuations in Fig. 2 cause the maximum Nash welfare to be 5. However, the only feasible allocation with a Nash welfare of 5 is $\langle\{1,3\},\{2\}\rangle$. This allocation is obviously not $\mathrm{EF} 1$, as agent 2 envies agent 1 , even after removing one item. EF1 allocations do exist for these instances, with allocations $\langle\{2\},\{1,3\}\rangle$ and $\langle\{1\},\{2,3\}\rangle$ being EF1 for $P_{3}$ and $\overline{P_{3}}$, respectively.

For any graph with both $G \neq K_{k}$ and $E \neq \emptyset$, we can use the valuations for two agents on $P_{3}$ and $\overline{P_{3}}$ to construct an instance where MNW does not result in EF1. First, let $n=\Delta(G)$ if $\Delta(G)+1=|M|$ and $n=\Delta(G)+1$ otherwise. Choose two agents and a set $S$ of three items, where the induced subgraph $G[S]$ is either $P_{3}$ or $\overline{P_{3}}$. Let the two agents value the items in $S$ as shown in Fig. 2, and let them value all other items at 0 . Since $G$ contains at least $\Delta(G)+1$ vertices and $|S|=3$, either $n=\Delta(G)=|M|-1$ and $|M \backslash S|=\Delta(G)+1-3=n-2$ or $n=\Delta(G)+1 \leq|M|-1$ and $|M \backslash S| \geq \Delta(G)+2-3=n-2$. Consequently, there is always at least $n-2$ items in $M \backslash S$. Let $S^{\prime}$ be a set of $n-2$ items from $M \backslash S$ and let each of the $n-2$ remaining agents assign a non-zero value to each item in $S^{\prime}$ and a value of 0 to the items in $M \backslash S^{\prime}$.

The MNW of the constructed instance is non-zero, as it is possible to allocate at least one item with non-zero value to each agent. For example, the items in $S$ can be allocated to the two agents that assign them a non-zero value, in a way that respects the item conflicts and gives each agent at least one item from $S$. The remaining $n-2$ agents can then be allocated one item each from $S^{\prime}$. When $n=\Delta(G)$, this is a feasible complete allocation, since $M=S \cup S^{\prime}$. When $n=\Delta(G)+1$, the instance can contain additional items that are all worth zero to all agents, i.e., $M \backslash\left(S \cup S^{\prime}\right) \neq \emptyset$. Since each item has at most $\Delta(G)$ conflicts, there exists for each item at least one agent that has not been allocated a conflicting item, no matter how the other items are allocated. Thus, any partial allocation that allocates all goods in $S$ and $S^{\prime}$ can trivially be extended to a complete allocation. Consequently, the MNW is only dependent on how $S$ and $S^{\prime}$ are allocated. Any allocation must allocate one item from $S^{\prime}$ to each of the $n-2$ agents in order to have a non-zero Nash welfare. Each item in $S$ must be given to one of the two agents that give them a non-zero value; otherwise, we could increase the Nash welfare by doing so. Thus, any MNW allocation must maximize the Nash welfare for the two agents on the induced subgraph $G[S]$, and, consequently, the allocation cannot be EF1. In the same fashion, a non-MNW, EF1 allocation may be constructed by allocating one item in $S^{\prime}$ to each of the $n-2$ agents and allocating 
the items in $S$ to the two agents in a way that neither envies the other after removing the other's most valuable item.

Proposition 6 has implications for fair allocation under cardinality constraints. Biswas and Barman have already pointed out that there exist instances of this problem where MNW does not lead to EF1 [4]. However, they do not provide any classification of such cases. By using similar valuations as those used to prove Proposition 6, one can construct examples whenever there are at least two categories, one of which contains two or more items and has a threshold of one. Then the same valuation function construction as for $\overline{P_{3}}$ can be used, picking two items from the category with a threshold of 1 and any item from another category.

While Proposition 6 shows that MNW does not lead to EF1 in general, it may still be possible that for some combinations of graphs, number of agents and restricted valuation functions, MNW does lead to EF1. Biswas and Barman [4] showed that for cardinality constraints (and other related matroid constraints), MNW leads to EF1 if all agents have identical valuations. As fair allocation of conflicting items is equivalent to cardinality constraints when all components are cliques, MNW leads to EF1 for instances with this class of conflict graph and identical valuations. ${ }^{6}$

\subsection{Proportionality}

While equitable coloring is equivalent to EF1 with identical values, the weighted version has not traditionally been a more general EF1 coloring, but is rather linked to the proportion any color receives of the total weight. That is, each node is weighted, and a weighted almost-equitable coloring is where each of the $n$ colors covers nodes with a total weight of at least $W / n$, where $W$ is the total of all the weights in the graph. This, of course, is equivalent to proportional allocation, where the agents have identical valuations (though the items do not necessarily have identical values). Some results apply even for general valuations (cf. Proposition 7).

Algorithm 1 This is the randomized coloring procedure with symmetry breaking, described by Pemmaraju and Srinivasan [30]. Specifically, it is the version that applies to weighted almost-equitable colorings.

1. Let $\pi$ be a permutation of $M$, selected uniformly at random.

2. Tentatively allocate each item to an agent, selected uniformly at random.

3. If an agent received conflicting items $j$ and $j^{\prime}$, where $\pi_{j}<\pi_{j^{\prime}}$, deallocate $j^{\prime}$.

4. Allocate remaining items arbitrarily, avoiding conflicts.

Note that in step 3, conflicts are based on the initial, tentative allocation, before any deallocations have taken place. Items $j$ and $j^{\prime}$ are considered to be in conflict even if $j$ has already been deallocated, so the order of deallocation is irrelevant.

\footnotetext{
${ }^{6}$ Note that this argument does not work for all the instances that were reduced to cardinality constraints in Proposition 2. When the components of the conflict graph are not cliques, there may be additional MNW allocations for the item conflict instance that are not EF1; indeed, an allocation that is MNW under cardinality constraints might conceivably, though feasible, not even be MNW under item conflicts.
} 
It should be obvious that this algorithm runs in polynomial time. What is more, it provides each agent with an expected fraction of $1-1 / e$, or almost $2 / 3$, of a proportional share.

Proposition 7 Given a problem instance $(N, M, V, G)$, where $|N|>\Delta(G)$, Algorithm 1 finds an approximate proportional allocation with item conflicts, where each agent can expect to get at least a fraction of $1-1$ /e of its share.

Proof Adapted from Lemma 5 of Pemmaraju and Srinivasan [30]. Specifically, their result uses exactly $\Delta(G)+1$ colors, whereas we need to permit any $n>\Delta(G)$. We wish to describe $p_{i j}$, the probability that item $j$ is allocated $i$ after step 3. (Note that this value will be identical for all $i$.) This will tell us the expected value of any bundle at that point, a value that can only increase in step 4 . That is, if we can show that $p_{i j} \geq(1-1 / e) / n$ for every item $j$, each bundle will—in expectation—be valued at no less than $(1-1 / e) \cdot v_{i}(M) / n$ by agent $i$. In other words, each agent can expect a fraction of $1-1 / e$ of its share.

The probability that $j$ is allocated to $i$ in step 2 is $1 / n$. If exactly $k$ of its neighbors are ranked earlier by $\pi$, the probability that $j$ is not removed again in step 3 is $(1-1 / n)^{k}$, meaning that we would have $p_{i j}=1 / n \cdot(1-1 / n)^{k}$. The probability of exactly $k$ neighbors are ranked before $j$ is $1 /(\delta(j)+1)$, where $\delta(j)$ is the degree of node $j$ in $G$, which gives us:

$$
\begin{aligned}
p_{i j} & =\frac{1}{n} \sum_{k=0}^{\delta(j)} \frac{1}{\delta(j)+1} \cdot\left(1-\frac{1}{n}\right)^{k}=\frac{1}{\delta(v)+1}\left(1-\left(1-\frac{1}{n}\right)^{\delta(v)+1}\right) \\
& \geq \frac{1}{n}\left(1-\left(1-\frac{1}{n}\right)^{n}\right) \geq \frac{1}{n}\left(1-\frac{1}{e}\right)
\end{aligned}
$$

This, then, produces the desired bound on the individual expectations.

Approximate proportionality implies approximate MMS, that is, if you receive a fraction $\alpha$ of your proportional share, you receive at least a fraction $\alpha$ of your maximin share. Note, however, that Proposition 7 only bounds the individual expectations. That is, this is not the expectation of the proportionality or MMS guarantee, i.e., the lowest fraction received by any agent.

The following result provides a deterministic bound for such a guarantee for proportionality, in a scenario where all agents have identical valuation functions. ${ }^{7}$

Proposition 8 For a problem instance $(N, M, V, G)$, where $|N|>\Delta(G)$, if agents have identical valuations $v: M \rightarrow[0,1]$, there exists an $\alpha$-approximate proportional allocation with

$$
\alpha \geq 1-1 / e-c n \sqrt{\ln (n) / W},
$$

for any $c>\sqrt{8}$, where $W=v(M)$.

\footnotetext{
7 In this case, MMS allocations are guaranteed to exist.
} 
Proof Based on Lemma 6 of Pemmaraju and Srinivasan [30], adapted from using exactly $\Delta(G)+1$ colors to permitting any $n>\Delta(G)$. Let $S$ be the bundle assigned to some agent by Algorithm 1. The strategy is to show that for any fixed $c>0$,

$$
\operatorname{Pr}\left[v(S)<\left(1-\frac{1}{e}\right) \cdot \frac{W}{n}-c \sqrt{W \ln n}\right] \leq \frac{1}{n^{c^{2} / 8}} .
$$

The probability that the leftmost inequality holds for at least one of the bundles is then, by the union bound, at most $n / n^{c^{2} / 8}=n^{1-c^{2} / 8}$. Choosing $c>\sqrt{8}$ leads to a positive probability that $n o$ agents receives less than $(1-1 / e) W / n-c \sqrt{W \ln n}$, which means that such an allocation must exist. Dividing by $W / n$ yields the approximation ratio $\alpha$.

For simplicity, we renumber the items according to $\pi$, and define $j \prec k$ to mean that $j<k$ and $j k \in E$. Let $a: M \rightarrow N$ represent the tentative allocation after step 2. We now consider differences in conditional expectations along $\pi$, for use in Azuma's martingale inequality:

$$
c_{j}=\left|\mathrm{E}\left[v(S) \mid a_{1}=i_{1}, \ldots, a_{j}=i_{j}\right]-\mathrm{E}\left[v(S) \mid a_{1}=i_{1}, \ldots, a_{j}=i_{j}^{\prime}\right]\right|
$$

There may be no difference (i.e., we may have $c_{j}=0$ ), as the change need not affect our bundle $S$. If, however, item $j$ is added or removed, an expected proportion of $1 / n$ of its lower-priority conflicting items will consequently be removed or added, respectively. Thus we can bound the differences by: ${ }^{8}$

$$
c_{j} \leq\left|v_{j}-\frac{1}{n} \sum_{j<k} v_{k}\right| \leq v_{j}+\frac{1}{n} \sum_{j<k} v_{k}
$$

Because values are in $[0,1]$, we have $c_{j} \leq 1+\Delta(G) / n<2$. We also have

$$
\sum_{j=1}^{m} c_{j} \leq \sum_{j=1}^{m} v_{j}+\frac{1}{n} \sum_{j=1}^{m} \sum_{j<k} v_{k} \leq W+\frac{\Delta(G)}{n} \cdot \sum_{j=1}^{m} v_{k}<2 W,
$$

and $\sum_{j=1}^{m} c_{j}^{2}<2 \sum_{j=1}^{m} c_{j}<4 W$. Azuma's inequality then produces

$$
\exp \left(\frac{-t^{2}}{2 \sum_{j=1}^{m} c_{j}^{2}}\right) \leq \exp \left(\frac{-c^{2} W \ln n}{8 W}\right)=\frac{1}{n^{c^{2} / 8}},
$$

which gives us (1).

While the bound given by Proposition 8 may be rather weak for many practical instances, it does show that, given the assumptions about $V$, for a given number of agents, the guaranteed fraction of proportionality improves as the number of items (or, rather, the sum of their values) grows. That is,

$$
\alpha(W)=1-1 / e-o(1),
$$

\footnotetext{
${ }^{8}$ Pemmaraju and Srinivasan have a factor of 2 rather than 1 in front of the second term, leading to $c>\sqrt{18}$, rather than $c>\sqrt{8}$ [30]. This is presumably to account for a fraction of $1 / n$ nodes lost as well as gained, leading to an overly conservative bound.
} 
where $W=v(M)$. (Pemmaraju and Srinivasan [30] also present two other similar guarantees, but they are given without proof, and so are not as straightforward to generalize to an arbitrary number of agents.)

\subsection{Maximin shares}

A common strategy for unconstrained MMS approximation is to use algorithms that build on the basic concept of bag filling, where items are placed in a bag one by one. If the bag reaches a certain value for some remaining agent, it is allocated as this agent's bundle. Then, the algorithm continues drawing items into a new bag, repeating the procedure until there are no remaining agents. It can easily be shown that a $(1-\alpha)$-MMS approximation can be achieved using this method when no item is worth more than $\alpha \mu_{i}$.

Bag filling is usually combined with preprocessing procedures, in order to reduce the impact of high-valued items on the achievable $\alpha$. A common procedure is to carefully construct a small set of high-valued items that combined are worth at least $\alpha \mu_{i}$ to some agent $i$. If the MMS of each agent is not reduced in the instance created by removing $i$ along with the items in the constructed set, i.e., assigning the set as $i$ 's bundle, then one can solve the problem for the reduced instance instead. A simple variant is to allocate individual items worth at least $\alpha \mu_{i}$. Other somewhat typical steps are conversion to socalled ordered instances [6] and creating initial bundles of high-valued items for the bag filling to fill with low-valued items [see, e.g., 15].

For instances with conflicting items, the conflict graph and especially its degree, $\Delta(G)$, limits the viability of these strategies. Most are simply not possible as they would cause the creation of infeasible bundles and their correctness proofs fail. Other strategies can be modified to work with the conflict graph, albeit at the cost of reduced efficiency. Bag filling is one of these, where instead of using $M$ as the source of items, we select one of a set of feasible bundles as the source for each bag to be filled, as described in the following algorithm.

Algorithm 2 Takes as input a problem instance $(N, M, V, G)$, a subset $N^{\prime} \subseteq N$, a feasible partition $A=\left\langle A_{1}, A_{2}, \ldots, A_{\ell}\right\rangle$ of some $M^{\prime} \subseteq M$, where $\ell \geq 1$, and limits $x_{i}>0$ for all $i \in N^{\prime}$. Repeating the following steps until no suitable $A_{k}$ is available will allocate bundles to some subset of agents $N^{\prime \prime} \subseteq N^{\prime}$, with each bundle worth at least $x_{i}$ to the agent $i$ that receives it.

1. Let $A_{k} \in A$ be a bundle with $v_{i}\left(A_{k}\right) \geq x_{i}$ for some $i \in N^{\prime}$.

2. Let $B$ be an empty bag.

3. Add items from $A_{k}$ to $B$, one at a time, until $v_{i}(B) \geq x_{i}$ for some $i \in N^{\prime}$.

4. Give $B$ to some $i \in N^{\prime}$ with $v_{i}(B) \geq x_{i}$, and let $N^{\prime}=N^{\prime} \backslash\{i\}$ and $A_{k}=A_{k} \backslash B$.

Separating the items into several feasible sets prevents the creation of unfeasible bundles. However, it will cause some items to stagnate, as some sets may be valued almost, but not quite, $x_{i}$ and will therefore not be used in the algorithm. Consequently, the guaranteed value each agent will receive is generally lower with multiple sets, if all agents are to receive a bundle. Lemma 3 provides a general formula to calculate the possible guarantees, which depends on the value of $M^{\prime}$, and the number of source bundles, $\ell$, and agents, $\left|N^{\prime}\right|$. 
Lemma 3 Given a problem instance $(N, M, V, G)$, a subset of agents $N^{\prime} \subseteq N$ and a feasible partition $A=\left\langle A_{1}, A_{2}, \ldots, A_{\ell}\right\rangle$ of some subset of items $M^{\prime} \subseteq M$, where $\ell \geq 1$, Algorithm 2 allocates a feasible bundle to each agent in $N^{\prime}$ if $v_{i j} \leq x_{i}$ and $x_{i} \leq v_{i}\left(M^{\prime}\right) /\left(\ell+2\left(\left|N^{\prime}\right|-1\right)\right)$ for all $j \in M^{\prime}, i \in N^{\prime}$. Items that are allocated are taken from at most $\min \left(\ell,\left|N^{\prime}\right|\right)$ sets in $A$ and the procedure runs in polynomial time.

Proof First, note that each allocated bundle consists of items taken from a single set $A_{k} \in A$. Since $A$ is feasible, each allocated bundle must be as well. Additionally, since each bundle is created from a single set in $A$, the algorithm cannot use items from more than $\left|N^{\prime}\right|$ sets in $A$, or $\ell$ sets if $\left|N^{\prime}\right|>\ell$. It is obvious that the procedure runs in polynomial time, as all operations can easily be verified to be performable in polynomial time in the number of agents and items.

If at all points during the execution of the algorithm, the remaining value in $A$, for any remaining agent, $i$, is no less than $\ell x_{i}$, there is always a bundle $A_{k} \in A$ with $v_{i}\left(A_{k}\right) \geq x_{i}$. Consequently, step 3 will at some point create a bundle worth $x_{i}$ to $i$, that is allocated to $i$ in step 4 . Note that since step 3 adds items to $B$ one at a time, when $v_{i j} \leq x_{i}$, each remaining agent, $i$, will value each already allocated bundle at no more than $2 x_{i}$. Otherwise, the value would already be at least $x_{i}$ before adding the last item. Hence, in the worst case, where $\left|N^{\prime}\right|-1$ agents have been allocated a bundle, the remaining agent $i$ will always value the remaining items at no less than $\ell x_{i}$ if

$$
\begin{aligned}
\ell x_{i} & \leq v_{i}(M)-\left(\left|N^{\prime}\right|-1\right) 2 x_{i} \\
x_{i} & \leq \frac{v_{i}(M)}{\ell+2\left(\left|N^{\prime}\right|-1\right)},
\end{aligned}
$$

which is the condition in the lemma.

The condition used in the proof of Lemma 3 where the remaining value is no less than $\ell x_{i}$, while sufficient, is overly strict for many inputs. In practice, one could, e.g., have that all remaining items are located in the same $A_{k} \in A$. In this case, it would be sufficient that the remaining value is no less than $x_{i}$. However, in the worst case, where the remaining value is spread evenly across $A$, then $\ell x_{i}$ is the minimum possible value for which the algorithm will allocate a bundle to each agent in $N^{\prime}$.

As with unconstrained bag filling, the guarantees of Algorithm 2 are dependent on the value of the highest-valued items. Thus, a way to handle high-valued items is needed. The standard method used without item conflicts, is to create a maximal matching between agents and items they value at least $\alpha \mu_{i}$. Allocating items based on this matching guarantees that a subset of agents each receives a one-item bundle valued at least $\alpha \mu_{i}$. In the reduced instance created by removing all matched agents and items, each agent has at least the same MMS as in the original instance and no items are worth $\alpha \mu_{i}$ or more. However, with a conflict graph, there are severe limitations on which allocations are feasible, and these are highly dependent on the number of agents. Consequently, a reduction using this type of matching of agent-item-pairs may lead either to a reduction in maximin shares or to the reduced instance becoming infeasible. Nonetheless, we can replicate some similar results when constrained by a conflict graph.

Lemma 4 For a problem instance $I=(N, M, V, G)$, with $|N|>\Delta(G)$, let $N^{\prime} \subseteq N$ and $M^{\prime} \subseteq M$, such that a perfect matching exists between the agents in $N^{\prime}$ and items they value 
at no less than $\alpha \mu_{i}^{I}$ in $M^{\prime}$. Let $I^{\prime}=\left(N \backslash N^{\prime}, M \backslash M^{\prime}, V^{\prime}, G^{\prime}\right)$, where $V^{\prime}=\left\{v_{i}: i \in N^{\prime}\right\}$ and $G^{\prime}=G\left[M \backslash M^{\prime}\right]$. Then,

(i) $v_{i}\left(M \backslash M^{\prime}\right) \geq\left|N \backslash N^{\prime}\right| \mu_{i}^{I}$ for all $i \in N$;

(ii) $\left|N \backslash N^{\prime}\right|>\Delta\left(G^{\prime}\right) \Longrightarrow \mu_{i}^{I^{\prime}} \geq \mu_{i}^{I}$ for all $i \in N \backslash N^{\prime}$; and

(iii) given a feasible partial allocation of $I^{\prime}$, where each agent $i \in N \backslash N^{\prime}$ receives at least $\alpha \mu_{i}^{I}$, a feasible complete $\alpha$-approximate MMS allocation of I can be constructed in polynomial time.

Proof Let $A=\left\langle A_{1}, A_{2}, \ldots, A_{|N|}\right\rangle$ be an MMS partition of the original instance for one of the agents, $i \in N$. After removing the items in $M^{\prime}$, at least $|N|-\left|M^{\prime}\right|=|N|-\left|N^{\prime}\right|=\left|N \backslash N^{\prime}\right|$ bundles in $A$ remain unchanged. Each unchanged bundle is valued at no less than $\mu_{i}^{I}$ by agent $i$. Consequently, $v_{i}\left(M \backslash M^{\prime}\right) \geq\left|N \backslash N^{\prime}\right| \mu_{i}^{I}$. Additionally, if $\left|N \backslash N^{\prime}\right|>\Delta\left(G^{\prime}\right)$, it is possible to chose $\left|N \backslash N^{\prime}\right|$ unchanged bundles in $A$ and reallocate the remaining items in the other bundles to these. This will be a feasible allocation of $I^{\prime}$, with each bundle worth at least $\mu_{i}^{I}$ to $i$, i.e., $\mu_{i}^{I^{\prime}} \geq \mu_{i}^{I}$.

For (iii), any perfect matching of the agents in $N^{\prime}$ to items in $M^{\prime}$ they value at no less than $\alpha \mu_{i}^{I}$ can be used to turn the feasible partial allocation of $I^{\prime}$ into a feasible partial $\alpha$ -approximate MMS allocation of $I$. The currently unallocated items can be arbitrarily allocated to agents that have no conflicting items, to create a complete $\alpha$-approximate MMS allocation. Since $|N|>\Delta(G)$, at least one such agent exists for each unallocated item. Both of these steps can easily be performed in polynomial time.

A matching of the type required for Lemma 4 can easily be found in the same way as in the unconstrained case. This is done by finding a maximum-cardinality matching in a bipartite graph of the agents and items, connecting each agent and item by an edge if the agent values the item at no less than $\alpha \mu_{i}{ }^{9}$ Thus, finding an $\alpha$-approximate MMS allocation is equivalent to finding a feasible partial allocation in the reduced instance, where each agent $i$ receives a bundle valued at least $\alpha \mu_{i}^{I}$. We can use Algorithm 2 on varying colorings of $G$ to achieve this, providing different guarantees for $\alpha$ depending on the conflict graph and the number of agents in the reduced instance.

Lemma 5 For a problem instance $(N, M, V, G)$, with $|N|>\Delta(G)$, let $n^{\prime}$ be the number of remaining agents after allocating items as described in Lemma 4 , so that $v_{i j} \leq \alpha \mu_{i}$ for all remaining agents and items. Then there exists a feasible $\alpha$-approximate MMS allocation if $\alpha$ is at most

$$
\begin{cases}1 & \text { if } n^{\prime} \leq 1, \\ n^{\prime} /\left(3 n^{\prime}-4\right) & \text { if } n^{\prime}=\Delta(G)+1=\chi(G), \\ n^{\prime} /\left(2 n^{\prime}+\chi(G)-3\right) & \text { if } n^{\prime} \geq \chi(G), \\ n^{\prime} /\left(3 n^{\prime}-3\right) & \text { if } n^{\prime}<\chi(G) .\end{cases}
$$

Proof Throughout the proof, assume that the valuations have been scaled so that $\mu_{i}=1$ for all agents $i$. As long as it can be shown that there exists a partial allocation where each

\footnotetext{
${ }^{9}$ It is not strictly necessary to find a maximum-cardinality matching - a maximal matching is all that is needed. In other words, one may arbitrarily allocate items one by one, to agents who value them at no less than $\alpha \mu_{i}$.
} 
agent in the reduced instance receives a bundle valued at least $\alpha$, then by Lemma 4 a feasible complete $\alpha$-approximate MMS allocation exists in the original instance. Note that Lemma 4 also guarantees that the items in the reduced instance are valued at no less than $n^{\prime}$ by each agent in the reduced instance.

Case $1\left(n^{\prime} \leq 1\right)$. The case of $n^{\prime}=0$ is trivial. If $n^{\prime}=1$, at least one bundle of any of the remaining agent's MMS partitions of the original instance remains unchanged after the reduction. Thus, the agent can be given this bundle valued at no less than 1 .

Case $2\left(n^{\prime} \geq \chi(G)\right)$. Create an MMS partition of the original instance for some agent $i$ in the reduced instance. Remove all items from the MMS partition that are not in the reduced instance and perform Algorithm 2 on the MMS partition with all remaining agents, never choosing $i$ when breaking a tie in step 4. Finally, create a $\chi(G)$-coloring of the remaining unallocated items in the reduced instance and perform Algorithm 2 on this coloring using the remaining agents. Agent $i$ is guaranteed to receive a bundle valued at $\alpha$ in the first bag filling if $\alpha \leq 1$, as there are at least $n^{\prime}$ bundles valued at no less than 1 in the MMS partition. Any agent that did not receive a bundle in the first bag filling valued the bundle of $i$ at less than $\alpha$. Thus, if there are $n^{\prime \prime}$ agents remaining after the first bag filling, it follows from Lemma 3 that all agents will receive a bundle in the second bag filling if:

$$
\begin{aligned}
\chi(G) \alpha & \leq n^{\prime}-\left(n^{\prime}-n^{\prime \prime}-1\right) 2 \alpha-\alpha-\left(n^{\prime \prime}-1\right) 2 \alpha \\
\chi(G) \alpha & \leq n^{\prime}-\left(n^{\prime}-2\right) 2 \alpha-\alpha \\
\alpha & \leq n^{\prime} /\left(2 n^{\prime}+\chi(G)-3\right)
\end{aligned}
$$

Case $3\left(\chi(G)>n^{\prime}\right)$. This case can be solved in a similar way to Case 2. However, instead of performing a second bag filling with a $\chi(G)$-coloring, choose an agent $i^{\prime}$ that did not receive a bundle in the first bag filling. For this agent, create an MMS partition of the original instance and remove all items that are currently allocated, i.e., let only unallocated items in the reduced instance remain. Perform Algorithm 2 on this partition using all remaining agents. Repeat this last bag filling step, exchanging $i^{\prime}$ with some remaining agent, until there are no remaining agents. As in Case 2, agent $i$ receives a bundle worth at least $\alpha$, when $\alpha \leq 1$. For the remaining agents, we require that they receive a bundle when Algorithm 2 is performed on their modified MMS partition. Lemma 4 guarantees that for any agent $i^{\prime}$, there are at least $n^{\prime}$ bundles in their MMS partition with a value of at least 1 each, before removing the allocated items in the reduced instance. Thus, it must be the case that after the earlier bag fillings, there remains enough value across these $n^{\prime}$ bundles that $i^{\prime}$ is guaranteed to receive a bundle in the bag filling on its modified MMS partition. By the same logic as in Case 2, this is by Lemma 3 guaranteed if:

$$
\begin{aligned}
n^{\prime} \alpha & \leq n^{\prime}-\left(n^{\prime}-2\right) 2 \alpha-\alpha \\
\alpha & \leq n^{\prime} /\left(3 n^{\prime}-3\right)
\end{aligned}
$$

Case $4\left(n^{\prime}=\Delta(G)+1=\chi(G)\right)$. Create an MMS partition of the reduced instance for some agent $i$ and perform Algorithm 2 on this partition with all other agents in the reduced instance. For these $n^{\prime}-1$ agents, we can choose an $\alpha=n^{\prime} /\left(3 n^{\prime}-4\right)$, by Lemma 3. Since there are $n^{\prime}$ bundles in the MMS partition and at most $n^{\prime}-1$ were used in the bag filling, there is at least one untouched bundle in the MMS partition and this can be given to $i$ guaranteeing a value of at least 1 . In this specific situation, this method is slightly better than Case 2. 
The minimum guaranteed value each agent will receive from Algorithm 2, is, as we have seen, highly dependent on the number of bundles in the supplied partition of $M^{\prime}$. The fewer bundles, the less value is required to guarantee the last agent a bundle and the more value can be given away to each agent. While it is never possible to partition $G$ into fewer than $\chi(G)$ feasible bundles, we saw in Lemma 5 that using specific partitions of $G$ provided a skewed distribution of the items across the bags, resulting in fewer wasted items. Lemma 5 does, however, not fully take into consideration the possibilities for reallocating items between bundles in order to slightly reduce the value of the unallocated items at the end of the bag filling. Depending on the situation, it may be possible to further limit the type of partition of $M^{\prime}$ used, or either combine or reorganize some of the bags during the execution of Algorithm 2, e.g., if the induced subgraph of $G$ over the remaining unallocated items can be colored with fewer than $\chi(G)$ colors. While we have not been able to find any general improvements, it is possible to use these insights to find slightly better guarantees when there are three agents.

Lemma 6 For a problem instance $(N, M, V, G)$, with $|N|=3>\Delta(G)$, there exists a 2/3-approximate MMS allocation.

Proof This result follows partially from Lemma 5. The lemma provides a guarantee of at least $2 / 3$ in all cases, except when both $n^{\prime}=3$ and $\chi(G) \in\{2,3\}$. These cases have the slightly worse guarantee of $3 / 5$ and must be handled differently. For the remainder of the proof, we assume that the values have been scaled so that for each agent $i, \mu_{i}=1$. Consequently, for each agent $i$, we have that $v_{i}(M) \geq 3$ and since $n^{\prime}=3$, all items are worth less than $2 / 3$.

Let $A=\left\langle A_{1}, A_{2}, A_{3}\right\rangle$ be an MMS partition for one of the agents. Let $i$ and $i^{\prime}$ be the two other agents. If there is a way to create bundles $B_{i}$ and $B_{i^{\prime}}$, worth at least $2 / 3$ to respectively $i$ and $i^{\prime}$ such that $\left(B_{i} \cup B_{i^{\prime}}\right) \subseteq\left(A_{k} \cup A_{k^{\prime}}\right)$, with $k, k^{\prime} \in\{1,2,3\}$, then a 2/3-approximate MMS allocation exists.

If there are two distinct bundles $A_{k}, A_{k^{\prime}} \in A$ such that $v_{i}\left(A_{k}\right) \geq 2 / 3$ and $v_{i^{\prime}}\left(A_{k^{\prime}}\right) \geq 2 / 3$, assigning $A_{k}$ to $i$ and $A_{k^{\prime}}$ to $i^{\prime}$ is sufficient for a 2/3-approximate MMS allocation. Otherwise, both $i$ and $i^{\prime}$ value only a single bundle $A_{k} \in A$ at $2 / 3$ or more. This bundle is then worth at least $5 / 3$ to both agents. We will show that it is always possible to partition $A_{k}$ together with possibly some items from another bundle $A_{k^{\prime}} \in A$, into two feasible bundles $B_{1}$ and $B_{2}$ both worth at least $2 / 3$ to $i$. Consequently, one of the bundles must be worth at least 5/6 to $i^{\prime}$ and a 2/3-approximate MMS allocation exists.

If $v_{i}\left(A_{k}\right) \geq 2$ or there is at most one item in $A_{k}$ valued more than $1 / 3$, then $A_{k}$ can be partitioned into two bundles worth at least $2 / 3$ by performing bag filling, always selecting the most valuable remaining item. Thus, assume that $5 / 3<v_{i}\left(A_{k}\right)<2$ and that the two most valuable items in $A_{k}, j$ and $j^{\prime}$, are such that $1 / 3<v_{i j^{\prime}} \leq v_{i j}<2 / 3$. Let $A_{k}^{\prime}=A_{k} \backslash\left\{j, j^{\prime}\right\}$. Then $v_{i}\left(A_{k}^{\prime}\right)>1 / 3$ and if $v_{i}\left(A_{k}^{\prime}\right) \geq 2 / 3$, then $\left\langle A_{k}^{\prime},\left\{j, j^{\prime}\right\}\right\rangle$ is a set of bundles that satisfies our requirements.

We now assume that $1 / 3<v_{i}\left(A_{k}^{\prime}\right)<2 / 3$, which implies that $v_{i j}>1 / 2$. Let $C_{j}$ and $C_{j^{\prime}}$ be the set of items in $M$ that conflict with, respectively, $j$ and $j^{\prime}$. If $v_{i}\left(C_{j}\right) \leq 2 / 3$, there must exist a set $A_{k^{\prime}} \in A \backslash\left\{A_{k}\right\}$, such that $v_{i}\left(A_{k^{\prime}} \backslash C_{j}\right) \geq 1 / 6$. In this case, $\left\langle\{j\} \cup\left(A_{k^{\prime}} \backslash C_{j}\right), A_{k} \backslash\{j\}\right\rangle$ is a set of bundles that satisfies our requirements. If $v_{i}\left(C_{j}\right)>2 / 3$, on the other hand, then because $\left|C_{j}\right| \leq \Delta(G)$, there must exist an item $j^{\prime \prime} \in C_{j}$, with $v_{i}\left(j^{\prime \prime}\right)>1 / 3$. Depending on whether $j^{\prime \prime}$ is in $C_{j^{\prime}}$ or not, one can combine either $A_{k}^{\prime}$ or $j^{\prime}$, respectively, with $j^{\prime \prime}$ to produce 
a bundle worth at least $2 / 3$, which can be complemented by a bundle of the other items in $A_{k}$.

Combining the results of Lemmas 4-6, it can be shown that any instance with $|N|>\Delta(G)$ has an $\alpha$-approximate MMS allocation with $\alpha>1 / 3$.

Theorem 1 For a problem instance $(N, M, V, G)$, with $|N|>\Delta(G)$, there exists an $\alpha$ -approximate MMS allocation, where $\alpha>1 / 3$. Specifically,

$$
\alpha= \begin{cases}1 & \text { if } n \leq 2, \\ \frac{2}{3} & \text { if } n=3 \\ \frac{n}{2 n-1} & \text { if } n \geq 4 \text { and } \chi(G)=2, \\ \frac{\chi(G)}{3 \chi(G)-3} & \text { if } n \geq 4 \text { and } \chi(G) \geq 3\end{cases}
$$

where $n=|N|$.

Proof We proceed by cases.

Case $1(n \leq 2)$. The case of $n=1$ is trivial. For $n=2$, this follows from Lemma 5. The same result could also be achieved by using the divide and choose protocol [31], which works in a similar way to the method in the lemma for $n=2$.

Case $2(n=3)$. Follows directly from Lemma 6.

In the remaining two cases, we use the fact that Lemmas 4 and 5 guarantee the existence of $\alpha$-approximate MMS allocations if $\alpha$ is not higher than the limits in Lemma 5. Since we cannot guarantee that a certain number of items are valued more than $\alpha$ in the general case, the highest value for $\alpha$ we may choose is the one that works for all $n^{\prime}$.

Case $3(n \geq 4$ and $\chi(G)=2)$. Either $n^{\prime}=1$ and $\alpha$ can be 1 , or $n^{\prime} \geq \chi(G)$ and we require $\alpha \leq n^{\prime} /\left(2 n^{\prime}-1\right)$. This is minimized when $n^{\prime}=n$ and thus $\alpha=n /(2 n-1)$ will work.

Case 4 ( $n \geq 4$ and $\chi(G) \geq 3$ ). Here, one can easily verify that the guarantee of Lemma 5 increases on the interval $[\chi(G), n]$ and decreases on the interval $[1, \chi(G)]$, if the improvements in the special case of $\chi(G)=\Delta(G)+1$ are ignored. Thus, the worst case, $n^{\prime}=\chi(G)$, allows for $\alpha=\chi(G) /(3 \chi(G)-3)$.

Note that Theorem 1 does not handle the case of $\chi(G)=\Delta(G)+1$ by itself, for which Lemma 5 provides better guarantees. Remark 2 shows the achievable guarantees in this case, which can be proven in a similar way to Theorem 1 .

Remark 2 For a problem instance $(N, M, V, G)$, with $|N|>\Delta(G),|N| \geq 4$ and $\Delta(G)+1=\chi(G)$, slightly better guarantees than in Theorem 1 can be found for $\Delta(G) \geq 2$ using Lemma 5. Specifically,

$$
\alpha=\left\{\begin{array}{l}
\frac{\chi(G)+1}{3 \chi(G)-1} \text { if } \chi(G)<7, \\
\frac{\chi(G)-1}{3 \chi(G)-6} \text { if } \chi(G) \geq 7 .
\end{array}\right.
$$

Theorem 1 shows the existence of $\alpha$-approximate MMS allocations with approximation factors better than $1 / 3$. The proof of the theorem is in fact constructive. However, the method used in the proof relies heavily on being able to both find a minimum vertex 
coloring of $G$ and being able to find MMS partitions for the agents. Both problems are NP-hard, leaving us without a polynomial-time approximation algorithm. Relaxing the approximation guarantees and employing some of the tricks used in MMS approximation when there are no restrictions on the bundles, it is possible to find a polynomialtime approximation algorithm for cases where $|N|>\Delta(G)$. Algorithm 3 outlines this algorithm.

Algorithm 3 Given the problem instance $(N, M, V, G)$, with $|N|>\Delta(G)$, find an $\alpha$ -approximate MMS allocation for sufficiently small $\alpha>0$.

1. Scale valuations so that $v_{i}(M)=|N|$ for all $i \in N$.

2. Repeatedly reduce the instance by allocating single items worth at least $\alpha$ to some remaining agent. Let $N^{\prime}$ and $M^{\prime}$ be the remaining agents and items at any stage, respectively. Rescale valuations to $v_{i}\left(M^{\prime}\right)=\left|N^{\prime}\right|$ for all $i \in M^{\prime}$ after each reduction.

3. (a) If $\left|N^{\prime}\right|=1$, give the remaining agent $i$ an approximate maximum weighted independent set on $G\left[M^{\prime}\right]$, weighted by $v_{i}$, using the approximation algorithm of Halldorsson and Lau [20].

(b) If $\left|N^{\prime}\right| \geq 2$ and $G\left[M^{\prime}\right]$ is $\Delta(G)$-colorable, perform Algorithm 2 on any $\Delta(G)$-coloring of $G\left[M^{\prime}\right]$.

(c) If $\Delta(G) \geq\left|N^{\prime}\right| \geq 2$ and $G\left[M^{\prime}\right]$ is not $\Delta(G)$-colorable, select an agent $i \in N^{\prime}$. Create a bundle $B$ of $i$ 's least-valued item in each non- $\Delta(G)$-colorable component of $G\left[M^{\prime}\right]$ and create a $\Delta(G)$-coloring of $G\left[M^{\prime} \backslash B\right]$. Scale the valuations of $i$ so that $v_{i}\left(M^{\prime} \backslash B\right)=\left|N^{\prime}\right|$, unless this results in $v_{i}\left(M^{\prime}\right)>\left|N^{\prime}\right|+1$, in which case rescale to $v_{i}\left(M^{\prime}\right)=\left|N^{\prime}\right|+1$. Perform Algorithm 2 on $B$ and the $\Delta(G)$-coloring, prioritizing other agents than $i$ in when breaking ties.

(d) If $\left|N^{\prime}\right|>\Delta(G) \geq 1$ and $G\left[M^{\prime}\right]$ is not $\Delta(G)$-colorable, perform Algorithm 2 on a $(\Delta(G)+1)$-coloring of $G\left[M^{\prime}\right]$.

4. Allocate remaining items without introducing conflicts in any bundle.

Algorithm 3 uses the same basic strategy as the approach in the existence proof, except that it relies on colorings that may be found in polynomial time. Note that instead of each special case in the bag filling step of the algorithm, one could use a $(\Delta(G)+1)$ -coloring of $G\left[M^{\prime}\right]$. This would, however, result in a reduction in the highest viable $\alpha$ by almost a factor of 2 in the worst case.

Theorem 2 For a problem instance $(N, M, V, G)$, with $|N|>\Delta(G)$, Algorithm 3 finds an $\alpha$-approximate MMS allocation in polynomial time, with $\alpha>1 / \Delta(G)$ when $\Delta(G)>2$. Specifically,

$$
\alpha= \begin{cases}1 / 2 & \text { if } \Delta(G)=1, \\ 3 / 7 & \text { if } \Delta(G)=2, \\ 2 /(\Delta(G)+2) & \text { if } \Delta(G)>2 .\end{cases}
$$

Proof In order for the algorithm to run in polynomial time, it cannot perform the NP-hard calculation of $\mu_{i}$. Instead, normalization is used, providing an upper bound on the value. 
This is done by continuously scaling the values of each agent $i$, so that $v_{i}\left(M^{\prime}\right)=\left|N^{\prime}\right|$ before, during and after step 2, where $M^{\prime}$ and $N^{\prime}$ are the remaining items and agents, respectively. By Lemmas 2 and 4 , this guarantees that $\mu_{i} \leq 1$ in all parts of the algorithm, except for agent $i$ in step 3c. The equivalent validity of the rescaling of agent $i$ 's valuations in step $3 \mathrm{c}$ will be covered later, together with the rest of the step.

In step 3a, all agents, except one, have received one-item bundles. This means that for the remaining agent, the allocated items can at most have been taken from $|N|-1$ of the $|N|$ bundles in any MMS partition of the original instance. Consequently, at least one of the bundles in each MMS partition exists in $G\left[M^{\prime}\right]$. Since a feasible bundle forms an independent set in the conflict graph, any maximum weighted independent set, $S$, of $G\left[M^{\prime}\right]$ weighted by this agent's valuations must fulfill $v_{i}(S) \geq \mu_{i}$. The approximation algorithm of Halldorsson and Lau [20] has an approximation factor of $3 /(\Delta(G)+2)$, which for all $\Delta(G) \geq 1$ is better than the limits of the theorem.

In step 3b, Lemma 3 guarantees that $\alpha$ can be $\left|N^{\prime}\right| /\left(2\left|N^{\prime}\right|+\Delta(G)-2\right)$, which when $\Delta(G) \leq 2$ is at least $1 / 2$ and otherwise minimized when $\left|N^{\prime}\right|=2$, i.e., $\alpha=2 /(\Delta(G)+2)$. Similarly, in step $3 \mathrm{~d}$, the function is minimized when $\left|N^{\prime}\right|=\Delta(G)+1$, allowing for $\alpha=(\Delta(G)+1) /(3 \Delta(G)+1)$, which is $1 / 2$ for $\Delta(G)=1,3 / 7$ for $\Delta(G)=2$ and otherwise at least $2 /(\Delta(G)+2)$, satisfying the guarantees of the theorem.

For step 3c we must show that (i) $G\left[M^{\prime} \backslash B\right]$ is $\Delta(G)$-colorable, (ii) the rescaling of agent $i$ 's valuation guarantees that $\mu_{i} \leq 1$, and (iii) each remaining agent receives a bundle that satisfies the requirements set forth in the theorem.

For (i), Brooks' theorem [10] tells us that each non- $\Delta(G)$-colorable component is either the complete graph of $\Delta(G)+1$ vertices, $K_{\Delta(G)+1}$, or, if $\Delta(G)=2$, a cycle of odd length, $C_{2 k+1}$. Removing a single item from the component will result in an induced subgraph of, respectively, $K_{\Delta(G)}$ and a path. Each of these can easily be verified to be $\Delta(G)$-colorable for $\Delta(G) \geq 2$. Subsequently, $G\left[M^{\prime} \backslash B\right]$ is $\Delta(G)$-colorable.

For the rescaling of agent $i$ 's valuations, (ii), let $A=\left\langle A_{1}, A_{2}, \ldots, A_{|N|}\right\rangle$ be any of $i$ 's MMS partitions of the original instance. Since $G\left[M^{\prime}\right]$ is not $\Delta(G)$-colorable, either a subset of the bundles in $A$ that contained items allocated in step 2 must contain an item from each non$\Delta(G)$-colorable component or at least $\Delta(G)+1 \geq\left|N^{\prime}\right|+1$ bundles remain untouched in step 2. In the first case, the remaining value is at least $\left|N^{\prime}\right| \mu_{i}+v_{i}(B)$, i.e., $v_{i}\left(M^{\prime} \backslash B\right) \leq\left|N^{\prime}\right|$ guarantees that $\mu_{i} \leq 1$. In the latter, the remaining value is at least $(\Delta(G)+1) \mu_{i}$, and $v_{i}\left(M^{\prime}\right) \leq\left|N^{\prime}\right|+1 \leq \Delta(G)+1$ guarantees that $\mu_{i} \leq 1$. Scaling the valuations to the minimum of these, guarantees that either the value of the bundles in the $\Delta(G)$-coloring is $\left|N^{\prime}\right|$ or the total value is $\left|N^{\prime}\right|+1$. In the first case, Lemma 3 allows $\alpha=2 /(\Delta(G)+2)$ for $i$, while in the latter it allows $\alpha=3 /(\Delta(G)+2)$ for $i$. In either case, agent $i$ 's bundle is compatible and $\alpha$ is at least as high as the one given in the theorem.

For the remaining agents, (iii), the bag filling algorithm is performed with $\Delta(G)+1$ bundles and a total value of $\left|N^{\prime}\right|$. This would by Lemma 3 result in $\alpha=2 /(\Delta(G)+3)$; however, since $i$ is never selected when breaking ties, either all other agents are given a bundle before $i$ or the bundle given to agent $i$ is worth less than $\alpha$. Consequently, we need to select $\alpha$ so that the following holds:

$$
\begin{aligned}
(\Delta(G)+1) \alpha & \leq\left|N^{\prime}\right|-2\left(\left|N^{\prime}\right|-2\right) \alpha-\alpha \\
\alpha & \leq 2 /(\Delta(G)+2)
\end{aligned}
$$

Lemma 4 guarantees that step 4 completes the $\alpha$-approximate MMS allocation.

It can easily be verified that steps $1,2,3 \mathrm{a}$ and 4 can all be completed in polynomial time in the number of agents and items. Checking that $G\left[M^{\prime}\right]$ is $\Delta(G)$-colorable is equivalent 
to checking if the induced subgraph contains either $K_{\Delta(G)+1}$ or if $\Delta(G)=2$ a component that forms an odd cycle. Each of these checks can be performed in polynomial time. A $(\Delta(G)+1)$-coloring can greedily be constructed in polynomial time and a polynomial-time algorithm of Lovász [27] can be used to construct a $\Delta(G)$-coloring in a $\Delta(G)$-colorable graph. Thus, steps $3 \mathrm{~b}, 3 \mathrm{c}$ and $3 \mathrm{~d}$ can be completed in polynomial time and the algorithm will find an $\alpha$-approximate MMS allocation in polynomial time.

Algorithm 3 relies on $(\Delta(G)+1)$ - and $\Delta(G)$-colorings in order to achieve its guarantee, as at least one of them can be constructed in polynomial time for any type of graph. Many graphs admit colorings using fewer colors. Optimal colorings can generally not be found in polynomial time. However, for some restricted graph classes we can find optimal colorings in polynomial time and for other there exists methods for finding colorings within a certain factor of optimum. For these classes, using the simpler colorings (i.e., with fewer colors) will improve the guarantees of Algorithm 2, which would improve the possible approximation factor that can be used in a polynomial-time algorithm. One such special case, is bipartite graphs, for which a 2-coloring can be found in polynomial time. Corollary 1 shows the possible approximation factor in this case, which is almost as good as our earlier theoretical lower bound.

Corollary 1 For a problem instance $(N, M, V, G)$, where $G$ is a bipartite graph and $|N|>\Delta(G)$, a 1/2-approximate MMS allocation can be found in polynomial time.

Proof When $G$ is bipartite, we do not need to rely on a $\Delta(G)$-coloring, as in Algorithm 3, in order to find an approximation in polynomial time. Instead, a 2-coloring can greedily be found in polynomial time. If the $\Delta(G)$-coloring is replaced by a 2-coloring, the proof of Theorem 2, without consideration for $\Delta(G)$-colorablility and $(\Delta(G)+1)$-colorings, guarantees an $\alpha$ of $1 / 2$.

While the use of better colorings can improve the value of $\alpha$ for specific graph classes, there are still quite strong limitations on how well Algorithm 2 can perform on a coloring of the graph. We will, for the straightforward approach used, be limited by an $\alpha$ of around $1 / \chi(G)$-and generally worse, because of the problems of finding a $\chi(G)$ -coloring. Using existing algorithms for more complex types of valuation functions with no conflicting items, we can guarantee a constant approximation factor in polynomial time for certain classes of graphs.

Proposition 9 For a problem instance $(N, M, V, G)$ with $|N|>\Delta(G)$, a 1/8-approximate MMS allocation may be found in polynomial time if the maximum weighted independent set problem can be solved in polynomial time on $G$ and all induced subgraphs of $G$.

Proof To find a polynomial-time algorithm, we will show a reduction to an unconstrained instance with fractionally subadditive (XOS) valuations. Ghodsi et al. [17] showed that for an instance of this type, a polynomial-time algorithm exists for 1/8-approximate MMS allocations given a polynomial-time demand oracle and XOS oracle.

Recall that a set function $f: 2^{M} \rightarrow \mathbb{R}_{\geq 0}$ is XOS if it can be represented as a finite set of additive functions $F=\left\{f_{1}, f_{2}, \ldots, f_{\ell}\right\}$, such that $f(X)$ is the maximum of all the additive functions applied to $X$, i.e., $f(X)=\max _{i=1}^{\ell} f_{i}(X)$. We will show that an oracle that finds the maximum weight $f(S)$ of an independent set in $S \subseteq M$ is XOS. For a graph $G$, let $\mathcal{I}(G)$ be 
the set of all the independent sets of $G$. For each $I \in \mathcal{I}(G)$, let $f_{I}: 2^{M} \rightarrow \mathbb{R}_{\geq 0}$ be an additive function, with values for $m \in M$ given by

$$
f_{I}(\{m\})= \begin{cases}w(m) & \text { if } m \in I \\ 0 & \text { otherwise }\end{cases}
$$

For a set of vertices $S \subseteq M, f_{I}$ finds the sum of the weight of the vertices in $S \cap I$. Let $F=\left\{f_{I}: I \in \mathcal{I}(G)\right\}$. Because $\mathcal{I}(G)$ contains all possible independent sets of $G$, the XOS function over $F, f^{\prime}$, will for any set of vertices $S \subseteq M$ be maximized by $f_{I}$ for any maximum weighted independent set, $I$, on $G[S]$. Thus, $f=f^{\prime}$ and the oracle is XOS. Consequently, XOS valuations may be calculated for each agent $i$ by using a maximum weighted independent set oracle on the graph using agent $i$ 's valuations as vertex weights.

We wish to use the 1/8-approximation algorithm for unconstrained MMS for instances with XOS valuations to find 1/8-approximate MMS allocations for instances with additive valuations and conflicting items. In order for this to be possible, we need to show that feasible allocations in either setting can be converted to feasible allocations in the other with at least the same value. If this is possible, then the MMS guarantee is the same in both settings and any $\alpha$-approximate MMS allocation in one setting can be converted to an $\alpha$ -approximate MMS allocation in the other setting.

Let $A=\left\langle A_{1}, A_{2}, \ldots, A_{|N|}\right\rangle$ be an allocation in the XOS setting. For each agent $i$, a maximum weighted independent set, $S_{i}$, of $A_{i}$, with respect to agent $i$ 's valuations, will have the same value as $A_{i}$. Consequently, $S=\left\langle S_{1}, S_{2}, \ldots, S_{|N|}\right\rangle$ is an allocation, possibly partial, where each agent receives the same value as in $A$. Since each $S_{i}$ forms an independent set in $G, S$ is also a feasible partial allocation with respect to the conflict graph, where each agent receives the same value as in the XOS setting. Without losing value, the unallocated items may be allocated to arbitrary agents without conflicts, as $|N|>\Delta(G)$. As a result, all allocations in the XOS setting can be converted to ones in the conflicting items setting with at least the same value.

Let $A=\left\langle A_{1}, A_{2}, \ldots, A_{|N|}\right\rangle$ be a feasible allocation in the conflicting items setting. Then each bundle $A_{i}$ forms an independent set of $G$ and $A_{i} \in \mathcal{I}$. Consequently, $f_{A_{i}}\left(A_{i}\right)=v_{i}\left(A_{i}\right)$ and bundle $A_{i}$ has at least the same value in the XOS setting.

In order for the 1/8-approximation algorithm of Ghodsi et al. to be usable in polynomial time, we must show that the reduction and subsequent conversion of the $\alpha$-approximate MMS allocation can be performed in polynomial time. The latter must be true, as it consists of solving the maximum independent set problem $|N|$ times in addition to reallocating the set of unallocated items, which can both be performed in polynomial time given our assumptions.

In order for the reduction to be performable in polynomial time, the demand oracle and XOS oracle used in the algorithm of Ghodsi et al. must both be creatable and queryable in polynomial time. The latter is an oracle that given a set of items, $S$, and the XOS function of an agent, $f_{i}$, provides a representation of the value each item in $S$ contributes in the $f \in F_{i}$ that is maximized for $S$. The construction of $f_{i}$ implies that $f=f_{I}$, where $I$ is a maximum weighted set of $G[S]$ with respect to agent $i$ 's valuations. An oracle that finds $I$ and provides a representation of $f_{I}$ is therefore an XOS oracle. Both of these operations can be performed in polynomial time, given our assumptions.

The demand oracle is an oracle that, given a list of prices $\left\langle p_{1}, p_{2}, \ldots, p_{m}\right\rangle$ and the XOS function of an agent, $f_{i}$, finds a set of items, $S$, that maximizes $f_{i}(S)-\sum_{s \in S} p_{s}$. In the algorithm, the prices are non-negative, which implies that $v_{i s}-p_{s} \leq v_{i s}$. Let $S^{\prime}$ be the possibly empty set of items, where $v_{i s}<p_{s}$. Then any maximum weighted independent 
set, $S$, in $G\left[M \backslash S^{\prime}\right]$ with weights $v_{i s}-p_{s}$ maximizes $f_{i}(S)-\sum_{s \in S} p_{s}$. To see that this is true, let $S$ be any set that maximizes the function. Any item in $S^{\prime}$ would provide a negative contribution and cannot be in $S$, i.e., $S \subseteq M \backslash S^{\prime}$. If $S$ is an independent set, then $f_{i}(S)-\sum_{s \in S} p_{s}=\sum_{s \in S}\left(v_{i s}-p_{s}\right)$, and consequently if $S$ is an independent set, it is a maximum weighted independent set in $G\left[M \backslash S^{\prime}\right]$ with weights $v_{i s}-p_{s}$. Now, we only need to show that for any maximum that is not an independent set, there exists an independent set with at least the same value. Assume that $S$ is not an independent set, and let $S^{*}$ be a maximum weighted independent set of $S$ with respect to the valuations of $i$. Then $f_{i}(S)=v_{i}\left(S^{*}\right)$ and $f_{i}(S)-\sum_{s \in S} p_{s} \leq f_{i}\left(S^{*}\right)-\sum_{s \in S^{*}} p_{s}$. Consequently, finding a maximum weighted independent set maximizes the function. This can be done in polynomial time and thus, the demand oracle can be queried in polynomial time.

The maximum weighted independent set problem is NP-hard, which limits the usefulness of Proposition 9. However, there are several graph classes where the problem is solvable in polynomial time; two noteworthy examples are bipartite graphs and claw-free graphs [29]. While we for bipartite graphs already know how to find 1/2-approximate MMS allocations in polynomial time, there is no restriction on the possible values for $\chi(G)$ in claw-free graphs. ${ }^{10}$ As a result, the reduction to XOS valuations could provide major improvements for dense, claw-free conflict graphs.

While we for some graphs cannot use the results of Proposition 9 to find 1/8-approximate MMS allocations in polynomial time, the reduction to XOS valuations holds nonetheless. This provides an alternative existence proof for MMS approximation, as Ghodsi et al. [17] showed the existence of 1/5-approximate MMS allocations for XOS valuations. Note that Ghodsi et al. also showed similar, but better, results for the more restrictive setting of submodular valuation functions. It might tempting to think that the maximum weighted independent set oracle is submodular; however, it is not, as is evident from Example 2.

Example 2 Consider a problem instance consisting of 4 items, where the valuation function of agent $i$ is given by $v_{i 1}=5, v_{i 2}=3, v_{i 3}=5, v_{i 4}=3$. Let the conflict graph be as follows:

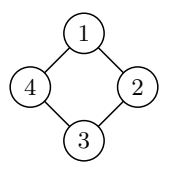

Let $f$ be the function that given a set of items finds the weight of the maximum weighted independent set using the valuations of the agent as vertex weights. If we let $X=\{1,2,4\}$ and $Y=\{2,3,4\}$, then $f(X)+f(Y)=12<16=f(X \cup Y)+f(X \cap Y)$. In other words, $f$ is not submodular.

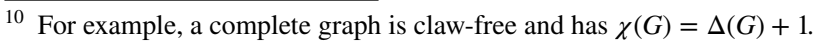


Table 1 The ranges of the randomly selected graph parameters

\begin{tabular}{llll}
\hline Par. & Range & Description & Model \\
\hline$n$ & $2,3, \ldots, 10$ & The number of agents & All \\
$m$ & $2 n, 2 n+1, \ldots, 4 n$ & The number of items (vertices) & All \\
$p$ & $(0,1)$ & Edge probability & Erdős-Rényi \\
$k$ & $1,2, \ldots, m$ & Initial size and connection degree & Barabási-Albert \\
$d$ & $2,4, \ldots, m / 2$ & Average degree & Watts-Strogatz \\
$\beta$ & {$[0,1)$} & Rewiring probability & Watts-Strogatz \\
\hline
\end{tabular}

\section{Conflicting items in practice}

We are interested in determining how fairness is affected, and the extent to which existing tools and formalisms still apply, with item conflicts. Specifically, when imposing item conflicts:

RQ1 To what extent do fair allocations (EF1, MMS) exist?

RQ2 How is the fairness (MMS, PROP) of random allocation affected?

RQ3 To what extent does MNW imply fairness (EF1, MMS)?

First of all, we wish to know to what extent fair allocation is actually possible in this new setting (RQ1). We know EF1 is not guaranteed in this setting, and that MMS may not be achievable even in the unconstrained case [23], but will the fairness guarantees go down to the point where these properties become the exception rather than the rule? And if MMS were to be unattainable for some instance, was it attainable without the item conflicts, or could the opposite be true, because of declining individual maximin shares?

We also wish to look at the prevalence of fair allocations (RQ2). With item conflicts, many allocations are no longer feasible, so utility will tend to decrease. However, whether fairness decreases is not a given. For example, although individual maximin shares will generally be lower in any setting with additional constraints, the degree of attainable MMS approximation may very well go up. And although one may lose the fairest allocations, it is quite possible that one loses many more unfair allocations; each item conflict, for example, prevents some degree of hoarding, as no agent can hold both items. To examine this effect empirically, we apply a random allocation procedure, as described in Proposition 7, studying the effect on the approximation of both MMS and proportionality, the latter to separate the effect of forced distribution of items from the lowering of individual maximin shares. This question is of interest in its own right, describing inherent properties of the problem. An answer might, however, also shed some light on the relative hardness of finding fair allocations, e.g., through randomized or heuristic procedures, with and without item conflicts.

Finally, we look at the impact on the maximum Nash welfare (MNW), inspired by the work of Caragiannis et al. (RQ3). They show that MNW is a useful tradeoff between efficiency and fairness, in the unconstrained allocation setting [12]. Is this still the case with item conflicts? For one thing, we look for a decrease in MNW, which might indicate either lower efficiency or fairness, or both. And while MNW still implies Pareto optimality when item conflicts are introduced, it no longer implies EF1 in general, and it is uncertain 
Table 2 Summary of the generated instances

\begin{tabular}{lccccccc}
\hline Type & Num. & MMS & $n$ & $m$ & $|E|$ & $\Delta$ & $\mathcal{C}$ \\
\hline Erdős-Rényi & 8620 & 8583 & 6.4 & 18.1 & 17.4 & 3.6 & 11.4 \\
Barabási-Albert & 5000 & 4956 & 7.7 & 19.8 & 20.5 & 6.0 & 19.8 \\
Watts-Strogatz & 5009 & 4975 & 7.1 & 20.1 & 30.7 & 4.4 & 19.4 \\
\hline
\end{tabular}

to which extent its approximation of MMS is preserved, so we explore the relationship between MNW and both of these properties.

\subsection{Experimental setup}

To address our empirical questions, we generated a collection of random instances, and found randomized allocations, MMS allocations, MNW allocations with and without EF1, all with and without item conflicts.

Problem instances. A central issue in generating instances is the choice of graph models. We selected three of the most popular and well-studied models of real-world graphs as our constraints:

(i) The Erdős-Rényi model, where each edge is present with probability $p$ [5];

(ii) The Barabási-Albert model, where a small graph is extended by preferential attachment [1]; and

(iii) The Watts-Strogatz model, where the edges of a regular ring lattice are randomly rewired with a certain probability [32].

Before producing an instance for a given graph type, all parameters were selected uniformly at random, in the ranges shown in Table 1 . The limits for $n$ and $m$ are based on real-world data from Caragiannis et al. [12], where the largest $n$ observed was 10, and the average ratio $m / n$ was approximately 3 . The upper limit for $d$ is based on the assumption of Watts and Strogatz that $d \ll m$ [32].

Once the parameters were set, a random graph $G$ was generated. If the graph had no edges, it was discarded, as this would merely be an instance of the ordinary allocation problem. ${ }^{11}$ If $\Delta(G) \geq n$, the graph was also discarded; for such instances, an allocation may not be feasible, and many results and methods, including the randomized algorithm of Proposition 7, do not apply. The process was repeated until we had 5000 graphs of each kind for which $n \leq \mathcal{C}(G)$. This was done to have enough data to study the prevalence of EF1 in cases where it is not immediately implied by Proposition 2. Finally, for each instance, a valuation was created by randomly dividing approximately 1000 points among the items, for each agent, in line with the value specification mechanism of Caragiannis et al. More specifically, each item was given a random real value, and the sum for each agent was scaled to 1000. Finally, individual values were rounded, as the mixed-integer program used to find MNW requires integral valuations [12].

Implementation The experiments were implemented in the Julia programming language, version 1.5.3 [3], using the LightGraphs package [9] for handling graphs, and the

11 This only applies to the Erdős-Rényi case. 

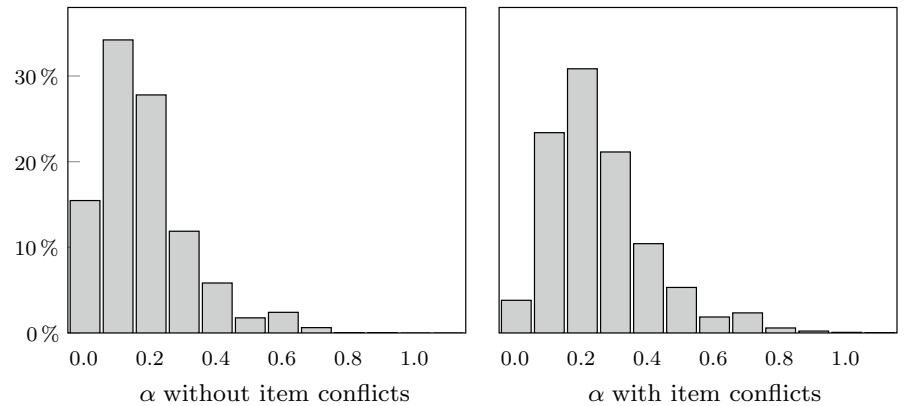

Fig. 3 The distribution of the approximation ratio $\alpha$ for MMS of a random distribution, with randomized tie breaking in the case of item conflicts (Algorithm 1). The bin labels indicate the lower inclusive limits. The item conflicts are seen to force a shift toward MMS

JuMP package [14] with Gurobi 9.1.1 [19] as the backend for solving mixed-integer linear programs (MIPs). ${ }^{12}$ For the randomized allocation, 1000 trials were performed for each instance, and the average recorded. Gurobi was run with a timeout of $5 \mathrm{~min}$ (on an 8-core Intel i9-9900K at $3.60 \mathrm{GHz}$ ).

For finding individual maximin shares, a straightforward maximin MIP was used, with $n$ clones of the given agent. The MMS allocations were then found by another maximin MIP, where agents' values were divided by their maximin shares. These maximin shares were also used to find the proportion of MMS for randomized allocation and MNW. The MNW allocations were found by a MIP based on the one described by Caragiannis et al. [12] (adapted to permit varying maximum bundle values), and MNW with EF1 was found by the same program, with added constraints requiring EF1. In all cases, item conflicts were handled by adding the necessary constraints to the relevant MIPs.

\subsection{Experimental results}

A summary of the instances is given in Table 2. While running the experiments, 115 instances $(0.006 \%)$ timed out when solving the mixed-integer programs for MMS, and were dropped from any MMS-based calculations. The number of remaining instances are listed in the MMS column. Following this are averages for the number of agents $(n)$, items $(m)$ and edges $(|E|)$, as well as for the maximum degree $(\Delta)$ and largest component $(\mathcal{C})$. Beyond timeouts, another issue is the required precision to compute $\mathrm{MNW}$, which may be quite high, even for a modest number of agents. When the precision is not available, the result may not be exactly MNW, but will still-with much laxer precision requirementsbe Pareto-optimal and, for the unconstrained case, EF1 [cf. 12]. For our instances, only $24.15 \%$ had the sufficient precision, and were thus guaranteed to find an MNW allocation, and not some close approximation. This means that our results may in some sense be seen as an empirical evaluation of the mixed-integer program of Caragiannis et al. [12] for finding MNW, rather than of the MNW itself. As this is currently the only feasible approach

\footnotetext{
12 The source code and raw experimental results are available as ancillary files for the preprint of this paper at https://arxiv.org/abs/2104.06280.
} 

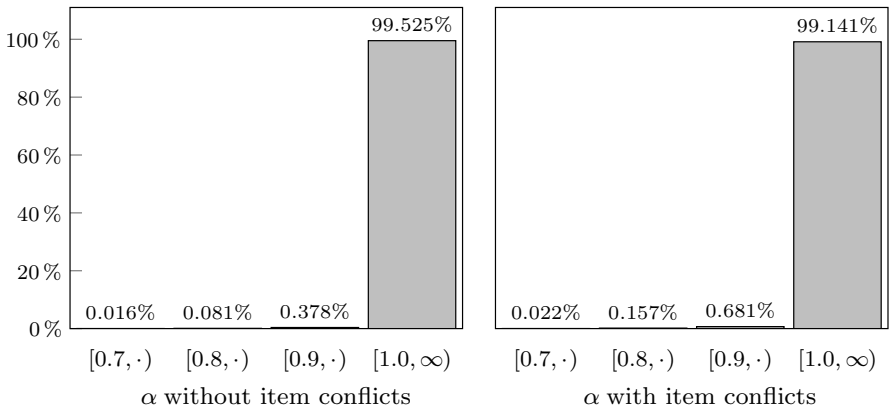

Fig. 4 The proportion of MMS attained by MNW before and after adding item conflicts

for finding MNW solutions, this still tells us something about the usefulness of MNW in practice. And although the solutions found might, in principle, lose some of the power of a guaranteed MNW solution, it seems unlikely that they would do better on any fairness criteria, at least in any systematic way; thus positive results could still be taken as support for the use of MNW.

The existence of fairness. Without constraints, EF1 allocations always exist, and all but the rarest instances have MMS allocations; we wish to know to what extent this holds true also under item conflicts (RQ1). Under item conflicts, we have established that EF1 is not guaranteed for any graph when $n \leq \Delta(G)$ (Proposition 1), and is otherwise guaranteed in some cases (Propositions 2, 4 and 5) but not in others (Proposition 3). The question is how common it is in practice. Similarly, we have shown that many approximations to MMS are guaranteed to exist, and although MMS is not guaranteed in general, might their existence still be the rule, despite having conflicting items? Our results are easily summed up in the affirmative: Every instance had both an EF1 allocation and an MMS allocation (also in the unconstrained case).

Expected fairness. When allocating items randomly to agents, one would expect a fairly even distribution, and introducing item conflicts does not affect this expectation too much on an individual level (cf. Proposition 7). We wish to examine the effects on the minimum as well, i.e., how closely we approximate MMS and proportionality (RQ2). For our random instances, random allocation disregarding the conflicts (i.e., a lottery) achieved, on average, an 0.23-approximate MMS allocation. With conflicts, however, we got an average approximation ratio of 0.30. A possible cause for the increase is that in the maximin problem being solved, values are normalized by the individual maximin shares of the agents, and these will generally go down with additional constraints. However, a similar increase is seen in the approximation ratio for proportionality (from 0.22 to 0.29 ), which would seem to indicate that the conflicts enforce a certain level of distribution of the items, with a forced increase in fairness. The distribution of approximation ratios for MMS is shown in Fig. 3.

The fairness of $M N W$. As opposed to the proportion of MMS for randomized allocation, the maximum Nash welfare will never increase when adding item conflicts, simply because the original is then a relaxation, with a higher possible optimum. The question is how much this impacts its usefulness as a tool for fair allocation (RQ3). For one thing, we know it can no longer guarantee EF1, even with $n>\Delta(G)$ (Proposition 6). It turns out, however, that it is still very close, with $99.88 \%$ of the MNW solutions for conflicting items being EF1. Conversely, adding a requirement of EF1 reduces the average MNW by $1.52 \%$. 
The reduction in proportion of MMS was not too substantial either. On average, the MNW solutions for our instances were 1.43-MMS, and adding item conflicts reduced this to 1.41-MMS (i.e., on average well above full MMS in both cases). The proportion of cases where MMS was achieved fell from 99.52 to $99.14 \%$, as illustrated in Fig. 4.

\section{Discussion}

The main purpose of this paper was to explore how introducing item conflicts affects the existence, prevalence and implications of fairly common fairness criteria. From a general theoretical standpoint, item conflicts seem to result in a certain reduction in fairness guarantees. At least, both the existence of EF1 and the guarantee that MNW leads to EF1 no longer hold. However, from a practical point of view, the reductions in guarantees seem to be minor and for some cases - non-existence of EF1—do not occur in our randomly generated instances.

It is interesting to see that while our theoretical results show that MNW no longer leads to EF1, in practice this only occurs in a handful of instances. Additionally, MNW seems to produce only slightly worse MMS approximations than in the unconstrained setting, still mostly resulting in MMS allocations. While no longer theoretically backed to the same extent as in the unconstrained setting, in practice MNW still seems to mostly provide similar benefits as those advocated by Caragiannis et al. [12], providing a practical tradeoff between efficiency and fairness.

For the non-existence of EF1, the experiments indicate that this most likely only very rarely occurs. This might to a certain degree be indicative of the extent to which there exist combinations of conflict graphs and number of agents such that we can construct instances without EF1 allocations. An insight that might help for the earlier mentioned open problem about which combinations of conflict graphs and the number of agents EF1 always exist for.

Both the non-existence proofs and the relative dearth of counter-examples in practice for both EF1 and the implication from MNW to EF1 tell us something about the prevalence of these properties. They do not, however, tell the full story about the probability of occurrence of non-existence. It might be that instances generated in our experiments provide types of instances where the non-existence is either likely or unlikely to occur in relation to the overall likelihood for all instances. An exploration of the probability with which each property is expected to occur would provide a better insight into the usefulness of the properties in real-world settings. Note that similar studies have been performed for envyfreeness in the unconstrained setting [see, e.g., 28].

For all but some special cases, there remains a large gap between our lower bound for the theoretical approximation guarantees of MMS and what our polynomial-time algorithms can guarantee. An interesting continuation of the research would be looking into improvements of the guarantees, both in general and for polynomial-time algorithms, especially considering that all instances in the experiments admit an MMS allocation. Conversely, it would not be unlikely that some, not too great, upper bound on polynomial approximation guarantees exist (unless $\mathrm{P}=\mathrm{NP}$ ), because of the close relation to hard graph problems and the upper bound for the closely related problem variant of Chiarelli et al. [13].

A natural extension to conflicting items that may be interesting to explore is introducing additional conflicts between agents and items, as in the standard weighted bipartite matching problem, where some agents simply cannot receive certain items. For example, this 
would be useful in the real-world example where items represent positions or roles in an organization. With agent-item conflicts, it would be possible to limit certain agents from taking on specific roles, either due to outside conflicts of interest or a lack of the require skillset needed for the role. In the same way that allocations for conflicting items form $n$-colorings of the conflict graph, the extension to item-agent conflicts would have allocations that form list colorings - a fairly well-known generalization of graph coloring, which has also been studied in the context of equitable coloring [22, 25].

Funding Open access funding provided by NTNU Norwegian University of Science and Technology (incl St. Olavs Hospital - Trondheim University Hospital).

\section{Declaration}

Conflict of interest The authors have no relevant financial or non-financial interests to disclose.

Open Access This article is licensed under a Creative Commons Attribution 4.0 International License, which permits use, sharing, adaptation, distribution and reproduction in any medium or format, as long as you give appropriate credit to the original author(s) and the source, provide a link to the Creative Commons licence, and indicate if changes were made. The images or other third party material in this article are included in the article's Creative Commons licence, unless indicated otherwise in a credit line to the material. If material is not included in the article's Creative Commons licence and your intended use is not permitted by statutory regulation or exceeds the permitted use, you will need to obtain permission directly from the copyright holder. To view a copy of this licence, visit http://creativecommons.org/licenses/by/4.0/.

\section{References}

1. Albert, R., \& Barabási, A. L. (2002). Statistical mechanics of complex networks. Reviews of modern physics, 74(1), 47.

2. Amanatidis, G., Markakis, E., Nikzad, A., \& Saberi, A. (2017). Approximation Algorithms for Computing Maximin Share Allocations. ACM Transactions on Algorithms, 13(4), 52:1-52:28. https://doi. org/10.1145/3147173.

3. Bezanson, J., Edelman, A., Karpinski, S., \& Shah, V. B. (2017). Julia: A fresh approach to numerical computing. SIAM review, 59(1), 65-98. https://doi.org/10.1137/141000671

4. Biswas, A., \& Barman, S. (2018). Fair division under cardinality constraints. In: Proceedings of the Twenty-Seventh International Joint Conference on Artificial Intelligence, International Joint Conferences on Artificial Intelligence Organization, pp 91-97

5. Bollobás, B. (2001). Random Graphs (2nd ed.). Cambridge University Press.

6. Bouveret, S., \& Lemaître, M. (2016). Characterizing conflicts in fair division of indivisible goods using a scale of criteria. Autonomous Agents and Multi-Agent Systems, 30(2), 259-290. https://doi.org/ 10.1007/s10458-015-9287-3

7. Bouveret, S., Cechlárová, K., Elkind, E., Igarashi, A., \& Peters, D. (2017). Fair division of a graph. In: Proceedings of the Twenty-Sixth International Joint Conference on Artificial Intelligence, International Joint Conferences on Artificial Intelligence Organization, Melbourne, Australia, pp 135-141, https:// doi.org/10.24963/ijcai.2017/20

8. Brandt, F., Conitzer, V., Endriss, U., Lang, J., \& Procaccia, A.D. (eds) (2016). Handbook of computational social choice. Cambridge University Press

9. Bromberger, S., \& Fairbanks, J. et al. (2017). JuliaGraphs/LightGraphs.jl: LightGraphs. https://doi.org/ 10.5281/zenodo.889971,

10. Brooks, R. L. (1941). On colouring the nodes of a network. Mathematical Proceedings of the Cambridge Philosophical Society, 37(2), 194-197. https://doi.org/10.1017/S030500410002168X, publisher: Cambridge University Press 
11. Budish, E. (2011). The Combinatorial Assignment Problem: Approximate Competitive Equilibrium from Equal Incomes. Journal of Political Economy, 119(6), 1061-1103. https://doi.org/10.1086/ 664613, publisher: The University of Chicago Press

12. Caragiannis, I., Kurokawa, D., Moulin, H., Procaccia, A. D., Shah, N., \& Wang, J. (2019). The unreasonable fairness of maximum nash welfare. ACM Transactions on Economics and Computation, 7(3), 12:1-12:32.

13. Chiarelli, N., Krnc, M., Milanič, M., Pferschy, U., Pivač, N., \& Schauer, J. (2020). Fair packing of independent sets. In: Combinatorial Algorithms, Springer International Publishing, Cham, Lecture Notes in Computer Science, pp 154-165, https://doi.org/10.1007/978-3-030-48966-3_12

14. Dunning, I., Huchette, J., \& Lubin, M. (2017). Jump: A modeling language for mathematical optimization. SIAM Review, 59(2), 295-320. https://doi.org/10.1137/15M1020575

15. Garg, J., \& Taki, S. (2020). An Improved Approximation Algorithm for Maximin Shares. In: Proceedings of the 21st ACM Conference on Economics and Computation, Association for Computing Machinery, New York, NY, USA, EC '20, pp 379-380, https://doi.org/10.1145/3391403.3399526

16. Garg, J., McGlaughlin, P., \& Taki, S. (2018). Approximating Maximin Share Allocations. In: Fineman JT, Mitzenmacher M (eds) 2nd Symposium on Simplicity in Algorithms (SOSA 2019), Schloss Dagstuhl-Leibniz-Zentrum fuer Informatik, Dagstuhl, Germany, OpenAccess Series in Informatics (OASIcs), vol 69, pp 20:1-20:11, https://doi.org/10.4230/OASIcs.SOSA.2019.20

17. Ghodsi, M., Hajiaghayi, M., Seddighin, M., Seddighin, S., \& Yami, H. (2018). Fair allocation of indivisible goods: Improvements and generalizations. In: Proceedings of the 2018 ACM Conference on Economics and Computation, Association for Computing Machinery, EC '18, pp 539-556, https://doi. org/10.1145/3219166.3219238

18. Gourvès, L., Monnot, J., \& Tlilane, L. (2013). A Protocol for Cutting Matroids Like Cakes. In: Chen Y, Immorlica N (eds) Web and Internet Economics, Springer, Berlin, Heidelberg, Lecture Notes in Computer Science, pp 216-229, https://doi.org/10.1007/978-3-642-45046-4_18

19. Gurobi Optimization L (2021). Gurobi optimizer reference manual. http://www.gurobi.com

20. Halldorsson, M. M., \& Lau, H. C. (1997). Low-degree graph partitioning via local search with applications to constraint satisfaction, max cut, and coloring. Journal of Graph Algorithms and Applications, 1(3), 1-13. https://doi.org/10.7155/jgaa.00003, https://ink.library.smu.edu.sg/sis_research/173

21. Kierstead, H. A., \& Kostochka, A. V. (2008). A short proof of the Hajnal-Szemerédi theorem on equitable colouring. Combinatorics, Probability and Computing, 17(2), 265-270. https://doi.org/10.1017/ S0963548307008619

22. Kostochka, A. V., Pelsmajer, M. J., \& West, D. B. (2003). A list analogue of equitable coloring. Journal of Graph Theory, 44(3), 166-177. https://doi.org/10.1002/jgt.10137

23. Kurokawa, D., Procaccia, A.D., \& Wang, J. (2016). When can the maximin share guarantee be guaranteed? In: Proceedings of the Thirtieth AAAI Conference on Artificial Intelligence, AAAI Press, Phoenix, Arizona, AAAI'16, pp 523-529

24. Lewis, RMR. (2016). A Guide to Graph Coloring. Springer International Publishing

25. Lih, K.W. (2013). Equitable coloring of graphs. In: Pardalos PM, Du DZ, Graham RL (eds) Handbook of Combinatorial Optimization, Springer, New York, NY, pp 1199-1248, https://doi.org/10.1007/9781-4419-7997-1_25

26. Lipton, R.J., Markakis, E., Mossel, E., \& Saberi, A. (2004). On approximately fair allocations of indivisible goods. In: Proceedings of the 5th ACM conference on Electronic commerce, Association for Computing Machinery, New York, NY, USA, EC '04, pp 125-131, https://doi.org/10.1145/988772. 988792

27. Lovász, L. (1975). Three short proofs in graph theory. Journal of Combinatorial Theory, Series B, 19(3), 269-271. https://doi.org/10.1016/0095-8956(75)90089-1

28. Manurangsi, P., \& Suksompong, W. (2018). When Do Envy-Free Allocations Exist? SIAM Journal on Discrete Mathematics, 34(3), 1505-1521. https://doi.org/10.1137/19M1279125

29. Minty, G. J. (1980). On maximal independent sets of vertices in claw-free graphs. Journal of Combinatorial Theory, Series B, 28(3), 284-304. https://doi.org/10.1016/0095-8956(80)90074-X

30. Pemmaraju, S., \& Srinivasan, A. (2008). The randomized coloring procedure with symmetry-breaking. In: Aceto L, Damgård I, Goldberg LA, Halldórsson MM, Ingólfsdóttir A, Walukiewicz I (eds) Automata, Languages and Programming, Springer, Berlin, Heidelberg, Lecture Notes in Computer Science, pp 306-319, https://doi.org/10.1007/978-3-540-70575-8_26

31. Steinhaus, H. (1948). The problem of fair division. Econometrica, 16(1), 101-104.

32. Watts, D. J., \& Strogatz, S. H. (1998). Collective dynamics of 'small-world' networks. Nature, 393(6684), 440-442. 
33. Woeginger, G. J. (1997). A polynomial-time approximation scheme for maximizing the minimum machine completion time. Operations Research Letters, 20(4), 149-154. https://doi.org/10.1016/ S0167-6377(96)00055-7

Publisher's Note Springer Nature remains neutral with regard to jurisdictional claims in published maps and institutional affiliations. 